\title{
Ação do Óxido Nitroso no Sistema Nervoso Central. Estudo Eletrofisiológico como Agente Único e como Agente Coadjuvante *
}

\section{Nitrous Oxide Action on the Central Nervous System. Electrophysiological Study as a Sole Agent or a Coadjuvant}

Verônica Vieira da Costa ${ }^{1}$, Renato Ângelo Saraiva, TSA $^{2}$

\begin{abstract}
RESUMO
Costa VV, Saraiva RA - Ação do Óxido Nitroso no Sistema Nervoso Central. Estudo Eletrofisiológico como Agente Único e como Agente Coadjuvante
\end{abstract}

Justificativa e Objetivos - O óxido nitroso é o agente anestésico inalatório mais utilizado em todo o mundo. Seu mecanismo de ação é bastante discutido, com base em resultados experimentais e em evidências clínicas. O objetivo deste estudo é avaliar a ação eletrofisiológica deste fármaco no Sistema Nervoso Central através de monitorização específica.

Método - Foram estudados vinte e cinco pacientes de ambos os sexos, com idades entre 6 e 25 anos, submetidos à cirurgia ortopédica ou plástica reparadora, os quais foram monitorizados com índice bispectral do eletroencefalograma (BIS) e potencial evocado somatossensitivo (PESS) durante a anestesia. Foram realizados registros basais do BIS e do PESS, bem como após o uso do óxido nitroso em fracionais alveolares (FA) de $30 \%, 50 \%$ e $66 \%$. Em seguida o óxido nitroso era descontinuado e administrado aleatoriamente isoflurano ou desflurano em 0,5 CAM e 1 CAM. Mantinha-se 1 CAM do determinado agente e o óxido nitroso era novamente administrado nas mesmas concentrações anteriores.

Resultados - O óxido nitroso quando utilizado como agente único, produz uma redução no BIS que, embora seja estatisticamente significante, não expressa um estado de hipnose. Esta redução também ocorre quando utilizado como agente coadjuvante mas sem importância clínica. Como agente único, o óxido nitroso deprimiu significantemente a amplitude das ondas cerebrais, sem promover aumento na latência. $O$ isoflurano e desflurano reduziram a amplitude e aumentaram a latência das ondas cerebrais. A associação do óxido nitroso a estes agentes, intensificou ainda mais estes efeitos nas ondas corticais. Não houve alteração significativa das ondas periférica e medular do PESS.

Conclusões - O óxido nitroso tem uma pequena ação hipnótica, que não é captada completamente pelo BIS. Tem ação acentuada nas estruturas corticais, tanto como agente

\footnotetext{
* Recebido do (Received from) Hospital Sarah Brasilia, DF

1. Anestesiologista do Hospital Sarah Brasília

2. Coordenador de Anestesiologia da Rede Sarah de Hospitais do ApareIho Locomotor
}

Apresentado (Submitted) em 15 de agosto de 2001

Aceito (Accepted) para publicação em 08 de novembro de 2001

Correspondência para (Mail to):

Dra. Verônica Vieira da Costa

Coordenação da Anestesiologia

SMHS Quadra 501 Conjunto: A

70335-901 Brasilia, DF

E-mail:veve@bsb.sarah.br

(C) Sociedade Brasileira de Anestesiologia, 2002 único como associado ao isoflurano e desflurano, o que pode explicar o seu bom efeito analgésico.

UNITERMOS - ANESTÉSICOS, Gasoso: óxido nitroso, Volátil: desflurano, isoflurano; MONITORIZAÇÃO: índice bispectral, potencial evocado somatossensitivo

\section{SUMMARY}

Costa VV, Saraiva RA - Nitrous Oxide Action on the Central Nervous System. Electrophysiological Study as a Sole Agent or a Coadjuvant

Background and Objectives - Nitrous oxide is the most widely used inhalational anesthetic worldwide. Its action mechanism is broadly discussed based on results of experimental studies and clinical evidences. The purpose of this study was to evaluate, through specific monitoring, nitrous oxide electrophysiological action on the central nervous system.

Methods - Twenty-five patients of both genders, aged 6 to 25 years, undergoing orthopedic or corrective plastic surgery, were monitored by electroencephalogram bispectral index (EEG-BIS) and somatosensory evoked potential (SEP) during anesthesia. BIS and SEP baseline values were recorded, as well as after fractional alveolar (FA) 30\%, 50\% and 66\% nitrous oxide administration. Then, nitrous oxide was withdrawn and isoflurane or desflurane were randomly administered in 0.5 and 1 MAC. While maintaining 1 MAC of one of those agents, nitrous oxide was again administered in the same previous concentrations.

Results - Nitrous oxide as sole agent caused a BIS decrease which, although statistically significant, did not represent a hypnotic state. This decrease was also observed when nitrous oxide was used as a coadjuvant agent, however without clinical significance. As sole agent, nitrous oxide significantly depressed brain waves amplitude, with no increase in onset time. Isoflurane and desflurane decreased the amplitude and increased onset time of brain waves. The association of nitrous oxide to those agents further increased these effects on cortical waves. There were no significant changes in peripheral and spinal cord SEP waves.

Conclusions - Nitrous oxide has a minor hypnotic action, which is not completely captured by EEG-BIS. It has a pronounced action on cortical structures, both as sole agent or associated to isoflurane or desflurane, which may explain its satisfactory analgesic effect.

KEY WORDS: ANESTHETICS, Gaseous: nitrous oxide, Volatile: desflurane, isoflurane; MONITORING: bispectral index, somatosensory evoked potentials

\section{INTRODUÇÃO}

óxido nitroso é um agente anestésico gasoso pouco potente. Sua concentração alveolar mínima (CAM) é $104 \%( \pm 10)$ de atmosfera que corresponde à pressão parcial 
de $805 \mathrm{mmHg}$ (nível do mar), só sendo obtida experimentalmente com câmara hiperbárica ${ }^{1}$, ou por estimativa de cálculo. É sempre usado em concentrações permissíveis (não hipóxicas), nunca acima de $70 \%$. Tem propriedade analgésica moderada, ação amnésica pouco pronunciada, pequeno poder imobilizante e efeito hipnótico muito leve ${ }^{2-4}$. Deste modo, compreende-se que suas indicações como agente anestésico único sejam muitíssimo limitadas, sendo mais utilizado como coadjuvante de anestésicos inalatórios mais potentes para reduzir as suas doses, e consequentemente seus efeitos colaterais.

Sua baixa solubilidade no sangue e nos tecidos orgânicos (coeficiente sangue/gás 0,47 e cérebro/sangue 1,1$)^{5}$ confere-Ihe propriedades farmacocinéticas muito especiais e desejáveis a um agente inalatório, especialmente como coadjuvante, considerando que sua captação e distribuição são muito rápidas assim como sua eliminação. Seu perfil farmacodinâmico aponta ações colaterais pouquíssimo pronunciadas, merecendo destaque as mínimas repercussões cardiovasculares e respiratórias. Além disso, é um gás pouco solúvel e com mínima metabolização. Sendo muito bem tolerado pelo organismo, é usado com grande freqüência ${ }^{6,7}$.

Tendo uma CAM muito próxima de $100 \%$, teoricamente, quando usado como coadjuvante reduziria a CAM do agente principal no valor da concentração que está sendo administrado. Por exemplo, tendo o halotano a CAM de $0,76 \%$, a adição de óxido nitroso à mistura inalatória na concentração de $50 \%$ reduziria esta CAM a $0,38 \%$.

De acordo com os relatos da literatura, esta relação se verifica quando associado a alguns agentes, não sendo no entanto uniforme para todos ${ }^{8}$. Além disto, alguns estudos eletroneurofisiológicos mostram a existência de relação linear deste efeito aditivo sobre determinado órgão do sistema nervoso central (SNC) ${ }^{9}$, não se observando, no entanto, em todos os órgãos do SNC ${ }^{10}$.

As características farmacológicas do óxido nitroso são muito discutidas. Não é considerado um anestésico completo, embora seja analgésico e amnésico. Não é hipnótico e pode ter ação hilariante. Pode produzir excitação atuando como depressor em áreas do SNC que têm função inibidora, liberando outras áreas que têm ação estimuladora, desencadeando uma reação tipicamente extrapiramidal. Seu mecanismo de ação sobre as estruturas do SNC não é totalmente conhecido. No entanto, muito já se sabe através de evidências encontradas em estudos experimentais e ensaios clínicos ${ }^{11,12}$. O óxido nitroso desenvolve estados clínicos bem característicos, diferentes dos demais anestésicos inalatórios. Provavelmente a avaliação eletrofisiológica poderá revelar resultados que mostram como é diferente a sua ação nas várias estruturas do SNC. O desafio de observar as alterações neurológicas funcionais durante a administração do óxido nitroso é uma motivação muito interessante. Assim sendo, este ensaio clínico foi desenvolvido para verificar de forma objetiva, através de monitorização específica, a ação deste fármaco sobre as estruturas do SNC, seja como agente único ou agente coadjuvante, correlacionando as variações encontradas com os seus efeitos farmacológicos.

\section{MÉTODO}

Após aprovação do Comitê de Ética do Hospital, foram estudados 25 pacientes com idade entre 6 e 25 anos, de ambos os sexos, classificados como estado físico ASA I e II, submetidos a cirurgias ortopédicas ou plásticas reparadoras de membros inferiores. Os pacientes receberam midazolam como medicação pré-anestésica na dose de $0,8 \mathrm{mg} \cdot \mathrm{kg}^{-1}$ via oral, 30 minutos antes da cirurgia, não ultrapassando a dose total de $15 \mathrm{mg}$. Na sala de indução anestésica foi iniciada a monitorização dos parâmetros fisiológicos habituais, bem como a monitorização eletroneurofisiológica.

Para monitorizar a ação sobre o córtex cerebral foi utilizado o eletroencefalograma com índice bispectral (BIS), sendo colocados os eletrodos tipo Zipprep (Aspect Medical System) na região frontal, com um eletrodo de cada lado e o terceiro de referência, situado entre eles. Foram portanto montados dois canais (Fp1e Fp2) mais um referencial (Fpz), de acordo com o Sistema Internacional 10-20 da Sociedade Internacional de Neurofisiologia Clínica (Figura 1), sendo analisados Fp1-Fpz no canal 1, Fp2-Fpz no canal 2, com o "terra" na região pré-auricular. $O$ índice bispectral (BIS) e as ondas dos dois canais de BIS foram registrados a partir de "épocas" sucessivas de 2 segundos de duração ${ }^{13}$ e atualizadas a cada cinco segundos, pelo monitor A 1000 (Aspects Medical Systems, Natick, MA). A banda de freqüência selecionada estava entre 0,5 e $30 \mathrm{Hertz}(\mathrm{Hz})$. A impedância foi verificada antes de cada aferição e manteve-se sempre inferior a 800 ohms $(\Omega)$. Foi utilizado sistema automático de detecção e rejeição de artefatos disponível no próprio monitor. O valor basal do BIS foi obtido antes da indução anestésica.

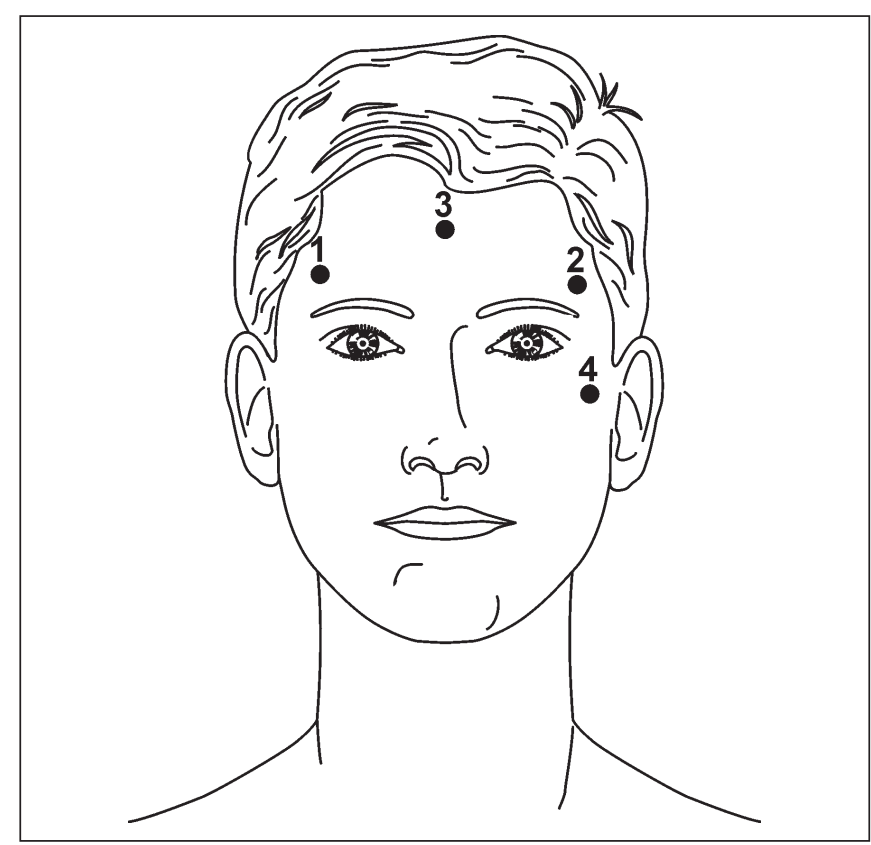

Figura 1 - Disposição dos Eletrodos do BIS de Acordo com o Sistema Internacional 10-20

Revista Brasileira de Anestesiologia Vol. 52, № 3, Maio - Junho, 2002 
Aanestesia foi induzida com propofol $\left(3 \mathrm{mg} \cdot \mathrm{kg}^{-1}\right)$ e vecurônio $\left(0,1 \mathrm{mg} \cdot \mathrm{kg}^{-1}\right)$, sendo em seguida realizada a intubação orotraqueal e iniciada a ventilação pulmonar mecânica. Todos os pacientes receberam anestesia peridural, no espaço entre a terceira e quarta vértebra lombar, com bupivacaína em concentração e volume que variaram com o peso do paciente, não ultrapassando a dose de $3 \mathrm{mg} \cdot \mathrm{kg}^{-1}$, com o objetivo de evitar a interferência de outros estímulos nos monitores utilizados. Trinta minutos após a administração do propofol foi realizado o registro do potencial evocado somatossensitivo (PESS), sendo este primeiro registro considerado valor basal, evitando-se assim artefatos gerados por tremores musculares, devido à baixa temperatura do centro cirúrgico. $\mathrm{O}$ monitor utilizado para realização do PESS foi o Aparelho Dantek - Keypoint versão 3.04, Denmark, a partir da estimulação do nervo mediano direito com eletrodo tipo barra, com distância catodo-anodo de $3,5 \mathrm{~cm}$. Os registros foram obtidos através de eletrodos de superfície nos seguintes locais: ponto de Erb, localizado na fossa supraclavicular direita (onda N9); sobre o processo espinhoso da segunda vértebra cervical (N13); no couro cabeludo, próximo ao córtex sensorial no lobo parietal, contralateral ao membro estimulado (ondas $\mathrm{N} 19$ e P22), de acordo com o Sistema Internacional 10-20. O eletrodo usado como referência ( $F z)$ foi colocado no centro da região frontal, próximo ao couro cabeludo, e o eletrodo "terra" foi fixado no ombro direito. A impedância foi mantida sempre inferior a $5 \mathrm{kohm}(\Omega)$. A intensidade do estímulo foi ajustada até se atingir o limiar de resposta motora, caracterizado pelo movimento do polegar (Thumb Twith). Para cada PESS, 20 impulsos de corrente constante foram repetidos com duração de 0,2 milissegundos ( $\mathrm{ms}$ ) e freqüência de descarga $5,1 \mathrm{~Hz}$. A banda de filtragem foi ajustada entre $20 \mathrm{~Hz}$ (baixa) e $3000 \mathrm{~Hz}$ (alta). As promediações (traçados) foram realizadas em duplicata, sendo comparadas as duas séries de registros para verificar sua reprodutibilidade. Foram avaliadas simultaneamente as ondas N9 (plexo braquial), N13 (medula espinhal cervical), N19 (tálamo-cortical) e P22 (cortical). A latência era registrada em milissegundos (ms) e a amplitude em microvolts ( $\mu \mathrm{v})$.

Os pacientes tiveram monitorização habitual das variáveis fisiológicas cardiorrespiratórias: eletrocardiograma (ECG), pressão arterial não invasiva (PA), saturação periférica de oxigênio da hemoglobina $\left(\mathrm{SpO}_{2}\right)$, pressão parcial de dióxido de carbono expirado final $\left(\mathrm{P}_{\mathrm{ET}} \mathrm{CO}_{2}\right)$, temperatura nasofaríngea, e concentração inspirada e expirada final (alveolar) dos agentes inalatórios utilizados, através do monitor de gases anestésicos. Após o registro basal do PESS, foi introduzido o óxido nitroso até se atingir uma concentração (fracional) alveolar (FA) de $30 \%$. Ao completar 5 minutos, era registrado novo PESS. Enquanto ocorriam as promediações do PESS, os valores do BIS que apareciam na tela do monitor eram anotados e depois calculada a média dos valores obtidos. Ao mesmo tempo eram anotados os valores das demais variáveis fisiológicas que estavam sendo avaliadas. A seguir, a concentração de óxido nitroso foi aumentada para 50 e $66 \%$, realizando-se o mesmo procedimento em relação aos registros das variáveis estudadas. Em seguida o óxido nitroso foi descontinuado e introduzido aleatoriamente isoflurano ou desflurano até ser atingida a FAde 0,5 CAM. Ao completar 10 minutos, novamente os registros de BIS, PESS e demais variáveis eram anotados. Ao aumentar a concentração alveolar para 1 CAM, novos registros e anotações eram feitos. Mantendo os pacientes com 1 CAM dos respectivos agentes, era reintroduzido o óxido nitroso nas mesmas concentrações anteriormente utilizadas (30, 50 e $66 \%)$ e os mesmos procedimentos eram realizadas nos intervalos de tempo previstos e na mesma seqüência.

$\mathrm{Na}$ análise estatística para os dados demográficos, foram utilizados os testes $t$ de Student e o Qui-quadrado. Para comparação dos valores das variáveis cardiovasculares, respiratórias e temperatura entre as diversas concentrações de óxido nitroso e outros agentes, foi utilizado o método de Bonferroni. Para verificar a significância das alterações das variáveis neurofisiológicas, foi realizada inicialmente análise exploratória de dados (cálculo de média e desvio padrão) e posteriormente utilizado método de medidas repetidas (teste de Fisher modificado), considerando estatisticamente significante o valor de $p \leq 0,05$.

\section{RESULTADOS}

Foram estudados 25 pacientes, que receberam inicialmente óxido nitroso, como agente único, sendo a seguir suspensa temporariamente a administração, quando 13 receberam isoflurano e 12 receberam desflurano, usando-se a seqüência descrita no método. Não houve diferença com relação ao sexo, idade, estado físico e peso entre o grupo de pacientes que recebeu óxido nitroso mais isoflurano e o grupo de pacientes que recebeu óxido nitroso e desflurano (Tabela I).

Tabela I - Dados Demográficos

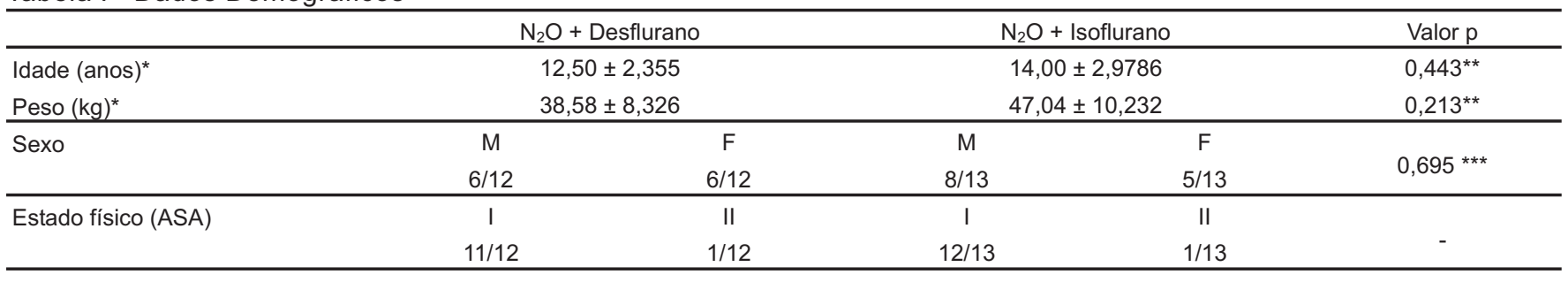

* Média $\pm 2 \mathrm{DP} ;{ }^{* *}$ Teste $t$ de Student; ${ }^{* * *}$ Teste Qui-quadrado 
Durante a administração de óxido nitroso nas várias concentrações, não houve alterações significativas nas variáveis fisiológicas estudadas. Quando foram introduzidos isoflurano e desflurano a estabilidade foi mantida, havendo no entanto, leve tendência a redução dos valores destas variáveis, mas sem significância estatística nem importância clínica. Os valores médios da $\mathrm{P}_{\mathrm{ET}} \mathrm{CO}_{2}$ tiveram variação entre 39 e 33 $\mathrm{mmHg}$, atingindo valores mais baixos nos pacientes que receberam isoflurano mas também sem significância estatística. Não houve alteração significativa da temperatura (Tabelas II e III).

Monitorização da Ação do Óxido Nitroso, Isoflurano e Desflurano sobre o Córtex Cerebral pelo EEG-BIS

O valor do BIS diminuiu em $23,8 \%$ quando o óxido nitroso foi utilizado como agente único na concentração de $30 \%$, mostrando significância estatística $(\mathrm{p}<0,001)$. À medida que a concentração aumentou para 50 e $66 \%$ houve apenas uma tendência muito discreta de aumento no valor do BIS, podendo-se admitir que manteve-se estável (Figura 2). Após 3 minutos da descontinuação do óxido nitroso, com sua FA próxima de zero, foi introduzido o isoflurano a 0,5 CAM, havendo queda de $42,6 \%$ do BIS em relação ao valor basal, estatisticamente significante $(p<0,001)$. Aumentando-se a concentração para 1 CAM, a redução aumentou para $66,17 \%$ do valor basal $(p<0,001)$.

Com o desflurano houve uma diminuição de $46,9 \%$ em relação ao valor basal com 0,5 CAM ( $p<0,001$ ) e 67,93\% quando a concentração aumentou para 1 CAM $(p<0,001)$.

Permanecendo a concentração de isoflurano em 1 CAM, e com a reintrodução do óxido nitroso em concentrações crescentes (30\%, 50\% e 66\%), houve pequena redução dos valo- res do BIS de $3,26 \%, 5,59 \%$ e $10,96 \%$ respectivamente. Com a adição de óxido nitroso a 1 CAM de desflurano, houve diminuição de $9,95 \%, 16,67 \%$ e 24,73\%, com 30, 50 e $66 \%$ de óxido nitroso respectivamente. A diminuição no valor do BIS foi maior naqueles pacientes nos quais o óxido nitroso foi associado ao desflurano em comparação àqueles em que o óxido nitroso foi associado ao isoflurano. A diminuição verificada no valor do BIS quando o isoflurano e desflurano foram utilizados sem adição de óxido nitroso foi súbita, mostrando uma curva quadrática (Figura 3). Quando o óxido nitroso foi associado a estes agentes mais potentes, a diminuição no valor no BIS foi mais suave, com tendência à estabilização a partir da concentração de $50 \%$.

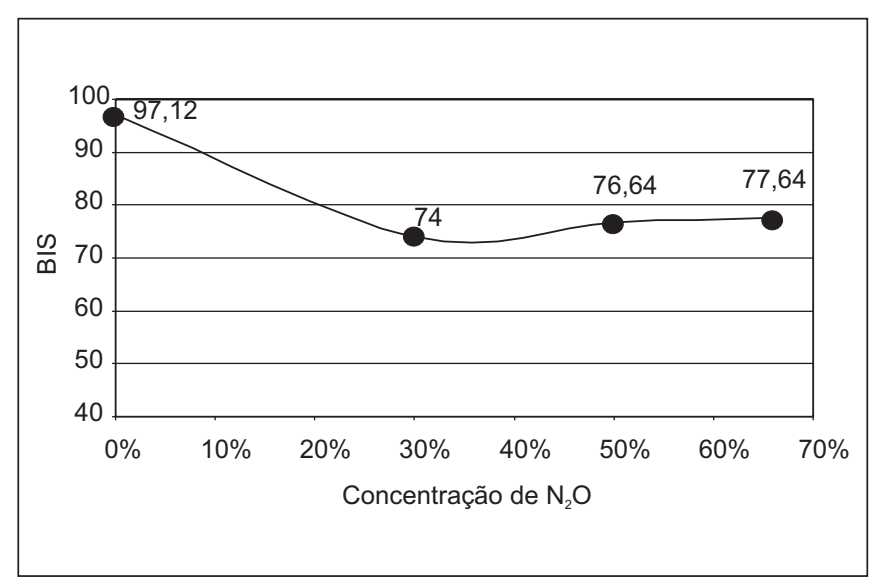

Figura 2 - Variação do Valor Médio do BIS com Óxido Nitroso $\left(\mathrm{N}_{2} \mathrm{O}\right)$ como Agente Único

( $p<0,001$ entre o valor basal e na FA $30 \%$ de óxido nitroso

Tabela II - Variáveis Fisiológicas Analisadas durante Administração de Óxido Nitroso como Agente Único

\begin{tabular}{lccccc}
\hline Concentração $\mathrm{N}_{2} \mathrm{O}$ & Pressão Arterial Média & Freqüência Cardíaca & Temperatura & $\mathrm{P}_{\mathrm{ETCO}}$ \\
\hline Basal & $91,35 \pm 11,86$ & $89,68 \pm 22,52$ & $36,22 \pm 1,14$ & - \\
$\mathrm{N}_{2} \mathrm{O} 30 \%$ & $98,76 \pm 17,61$ & $107,12 \pm 20,57$ & $36,11 \pm 1,04$ & $39,04 \pm 1,04$ \\
$\mathrm{~N}_{2} \mathrm{O} 50 \%$ & $101,73 \pm 14,46$ & $103,16 \pm 22,72$ & $36,08 \pm 1,15$ & $99 \pm 5,87$ \\
$\mathrm{~N}_{2} \mathrm{O} 66 \%$ & $96,11 \pm 15,63$ & $96,92 \pm 23,70$ & $36,01 \pm 1,10$ & $39,64 \pm 4,73$ & $38,84 \pm 4,95$ \\
\hline
\end{tabular}

* Valores expressos em Média $\pm \mathrm{DP}$

Tabela III - Variáveis Fisiológicas Analisadas Durante Administração de Óxido Nitroso Associado a Isoflurano e Desflurano

\begin{tabular}{|c|c|c|c|c|c|c|}
\hline Concentração $\mathrm{N}_{2} \mathrm{O}$ & Anestésico & Pressão Arterial Média & Freqüência Cardíaca & Temperatura & $\mathrm{P}_{\mathrm{ET}} \mathrm{CO}_{2}$ & $\mathrm{SpO}_{2}$ \\
\hline \multirow[t]{2}{*}{$1 \mathrm{CAM}$} & Isoflurano & $74,31 \pm 15,63$ & $80,92 \pm 22,6$ & $35,53 \pm 1,28$ & $34,08 \pm 3,28$ & $99,62 \pm 0,65$ \\
\hline & Desflurano & $75,67 \pm 17,87$ & $89,25 \pm 25,11$ & $35,96 \pm 0,74$ & $36,00 \pm 2,22$ & $99,50 \pm 0,52$ \\
\hline $1 \mathrm{CAM}+\mathrm{N}_{2} \mathrm{O} 30 \%$ & Desflurano & $68,33 \pm 8,89$ & $84,00 \pm 21,59$ & $35,91 \pm 0,67$ & $38,00 \pm 2,37$ & $99,33 \pm 0,49$ \\
\hline \multirow[t]{2}{*}{$1 \mathrm{CAM}+\mathrm{N}_{2} \mathrm{O} 50 \%$} & Isoflurano & $65,23 \pm 7,11$ & $75,62 \pm 19,89$ & $35,21 \pm 1,32$ & $34,46 \pm 2,76$ & $99,46 \pm 0,78$ \\
\hline & Desflurano & $69,13 \pm 8,24$ & $83,00 \pm 21,15$ & $35,88 \pm 0,68$ & $38,25 \pm 2,77$ & $99,00 \pm 0,6$ \\
\hline \multirow[t]{2}{*}{$1 \mathrm{CAM}+\mathrm{N}_{2} \mathrm{O} 66 \%$} & Isoflurano & $64,54 \pm 7,43$ & $74,08 \pm 19,96$ & $35,07 \pm 1,23$ & $33,85 \pm 2,88$ & $99,00 \pm 0,91$ \\
\hline & Desflurano & $65,58 \pm 7,54$ & $83,75 \pm 19,92$ & $35,72 \pm 0,6$ & $37,08 \pm 4,83$ & $98,67 \pm 1,07$ \\
\hline
\end{tabular}

* Valores expressos em Média \pm DP 


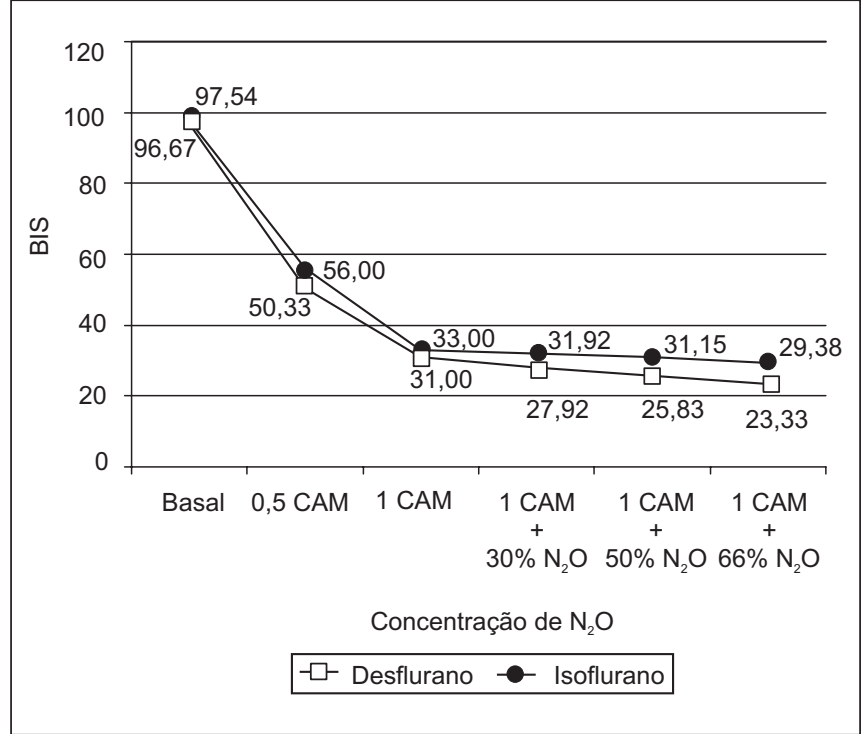

Figura 3 - Variação do Valor do BIS com Óxido Nitroso associado a Isoflurano e Desflurano

( $p<0,001$ entre o valor basal e FA de 0,5 CAM e entre o valor basal de FA 1 CAM)

Monitorização sobre a Ação do Óxido Nitroso, Isoflurano e Desflurano sobre Nervo Periférico, Medula Espinhal e Cérebro através da Evocação do Estímulo Nervoso Periférico PESS

Ação sobre a estrutura nervosa periférica (plexo braquial), onda N9 do PESS:

O óxido nitroso como agente único não alterou a amplitude e a latência nas fracionais alveolares (FA) de 30,50 e $66 \%$. O isoflurano e o desflurano também não alteraram a amplitude e a latência quando administrados em FAde 0,5e 1 CAM. A associação do óxido nitroso em FA de 30,50 e $66 \%$ ao isoflurano e desflurano em FA de 1 CAM, também não alterou a amplitude e a latência da onda N9.

Ação sobre a estrutura da medula espinhal, onda N13 do PESS:

Também não houve alterações significativas na amplitude e latência com o uso de óxido nitroso em FA de 30, 50 e 66\%, e de isoflurano ou desflurano a 0,5 e 1 CAM.

Ação sobre a estrutura tálamo-cerebral, onda N19:

O óxido nitroso reduziu sua amplitude em 10,76\% com FA $30 \%$ em relação ao valor basal. Com FA $50 \%$ reduziu em $29,57 \%$ e com FA $66 \%$ reduziu $28,09 \%(p<0,001)$. Não houve alteração na latência com as concentrações utilizadas (Figuras 4 e 5 ).

O isoflurano promoveu redução na amplitude em 18,93\% a 0,5 CAM $(p=0,303)$ e $16,18 \% \%$ a 1 CAM $(p=0,42)$ em relação ao valor basal. Alatência aumentou 3,72\% com 0,5 CAM e $11,84 \%$ com 1 CAM. Estas alterações, no entanto, não tiveram significância estatística.

Revista Brasileira de Anestesiologia

Vol. 52, № 3, Maio - Junho, 2002

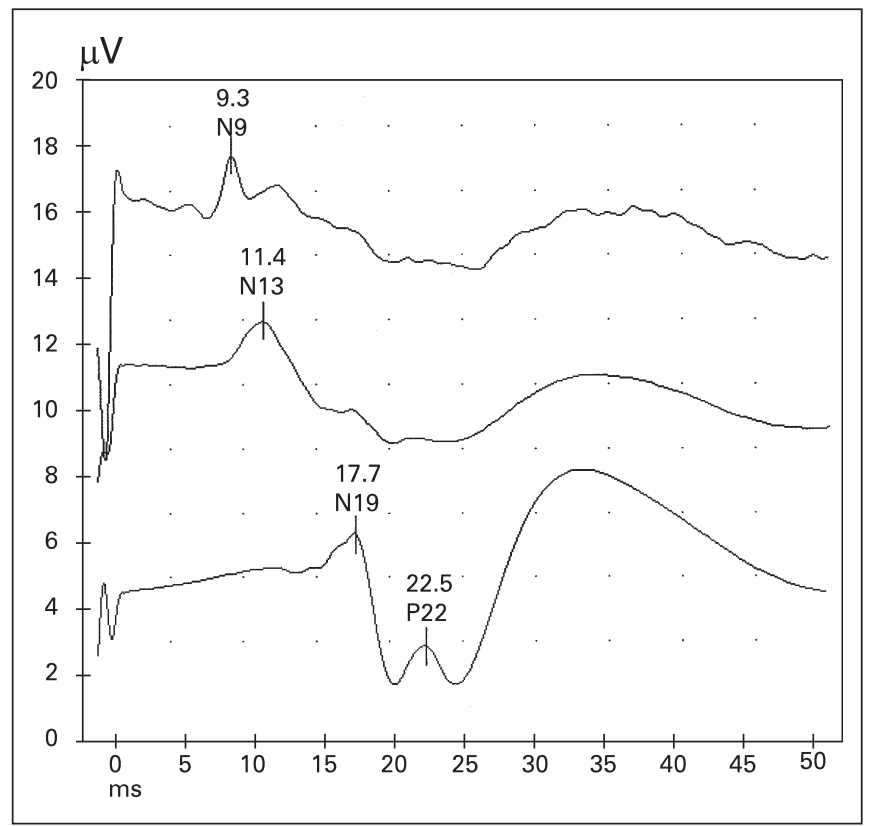

Figura 4 - Ondas do PESS (N9, N13, N19, P22) Valor Basal (antes da Administração do Óxido Nitroso)

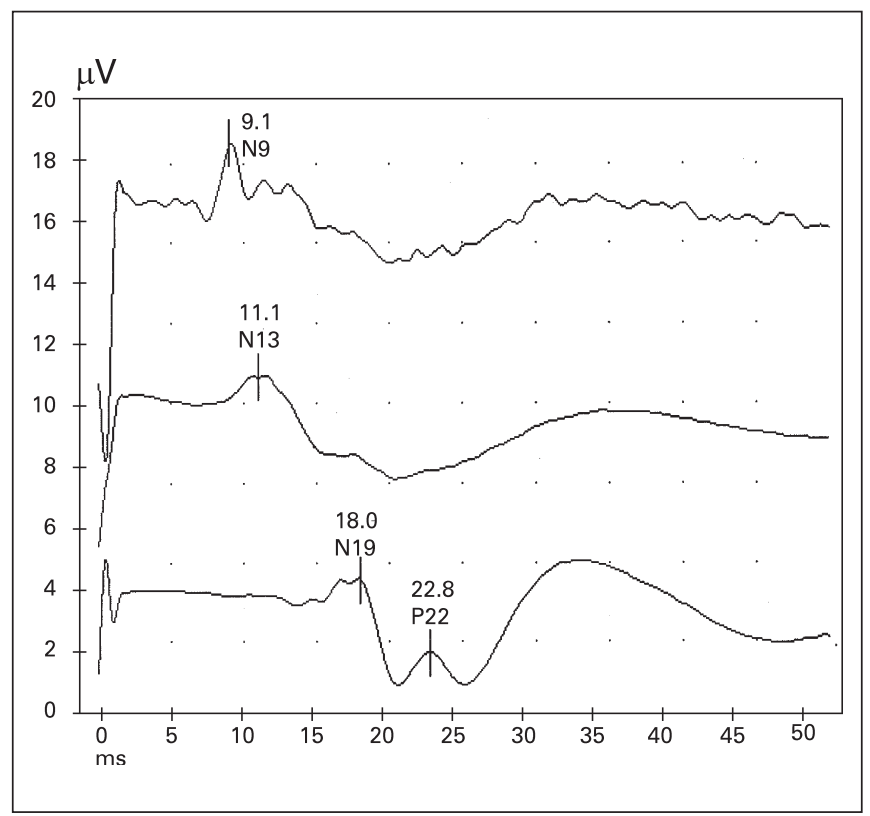

Figura 5 - Ondas do PESS (N9, N13, N19, P22). Paciente com FA de Óxido Nitroso $66 \%$. Verifica-se uma Pequena Redução na Amplitude da N13 e uma Redução Maior na Amplitude N19 e P22

O desflurano reduziu a amplitude em $19,21 \%$ a 0,5 CAM $(p=$ $0,37)$ e $20 \%$ a 1 CAM $(p=0,42)$ em relação ao valor basal. A latência aumentou $5,90 \%$ a 0,5 CAM e $14,60 \%$ a 1 CAM em relação ao valor basal. Estas alterações também não tiveram significância estatística.

Aassociação de isoflurano a 1 CAM e óxido nitroso com FAde 30,50 e $66 \%$ reduziu ainda mais a amplitude, sendo esta re- 
dução, em valores percentuais, de 29,92\% ( $p=0,137)$, $37,64 \%(p=0,067)$ e $58,20 \%(p=0,014)$ respectivamente. $O$ aumento da latência não foi intensificado.

A associação de desflurano a 1 CAM e óxido nitroso com FA de 30,50 e $66 \%$ reduziu ainda mais a amplitude, sendo esta redução de $48,72 \%(p=0,005), 51,36 \%(p=0,024)$ e $60,50 \%$ $(p=0,010)$ respectivamente. O aumento da latência foi intensificado quando o óxido nitroso foi associado com FA $66 \%$ $(20,13 \%$ de aumento), sem no entanto apresentar significância estatística $(p=0,24)$.

Ação sobre a estrutura cerebral, onda P22:

O óxido nitroso reduziu sua amplitude em $35,54 \%$ com FA $30 \%, 38,52 \%$ em FA $50 \%$ e $46,48 \%$ com FA $66 \%$ em relação ao valor basal $(p<0,001)$. Não houve alteração na latência com as concentrações utilizadas (Figura 6).

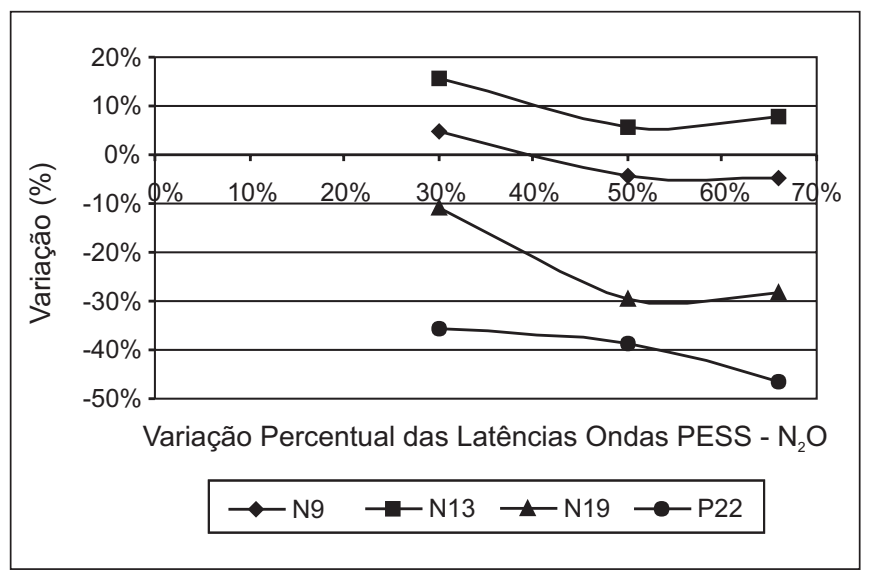

Figura 6 - Variação Percentual da Amplitude das Ondas do PESS com Óxido Nitroso Administrado como Agente Único $(p>$ 0,001 . Comparado entre o Valor Basal e após Administração de $\mathrm{N}_{2} \mathrm{O}$ a 30,50 e $66 \%$

O isoflurano a 0,5 CAM reduziu a amplitude em 18,81\% em relação ao valor basal e quando foi administrado em FA de 1 CAM reduziu a amplitude em $58,59 \%$, sendo estas alterações estatisticamente significantes ( $p=0,017$ e $p=0,014$ respectivamente). Também aumentou a latência em 4,78\% com a FA de 0,5 CAM e em 14,14\% com FA de 1 CAM em relação ao valor basal. O aumento da latência não foi estatisticamente significativo.

O desflurano reduziu a amplitude em $34,13 \% \%$ a 0,5 CAM e em $67,69 \%$ a 1 CAM em relação ao valor basal, apresentando siginificância estatística quando foi administrado com FAde 1 CAM $(p=0,001)$. Ao mesmo tempo aumentou a latência em $7,48 \%$ e 15,99\% com a FAde 0,5 CAM e 1 CAM respectivamente, em relação ao valor basal, sem haver significância estatística.

Aassociação de isoflurano a 1 CAM e óxido nitroso a FA $30 \%$, $50 \%$ e $66 \%$ reduziu ainda mais a amplitude em $7,16 \%(p=$ $0,15), 54,58 \%(p<0,06)$ e 41,80\% ( $p<0,001)$, respectivamente, sendo estas reduções estatisticamente significantes na concentração alveolar de $66 \%$. O aumento na latência foi intensificado com FA de 30, 50 e 66\% em 20,47\% ( $p=0,043)$, $22,05 \%(p=0,013)$ e $27,24 \%(p=0,066)$ respectivamente (Figuras 7 e 8 ).

A associação de desflurano a 1 CAM e óxido nitroso com FA $30 \%, 50 \%$ e $66 \%$ reduziu a amplitude em $27,23 \%(p=0,03)$, $65,65 \%(0,05)$ e $67,71 \%(p=0,04)$ respectivamente. $\mathrm{O}$ aumento da latência foi intensificado em $23,91 \%(p=0,005)$, $27,25 \%(p=0,007)$ e $31,33 \%(p<0,009)$ nas FA de 30,50 e $66 \%$ respectivamente (Figuras 9 e 10).

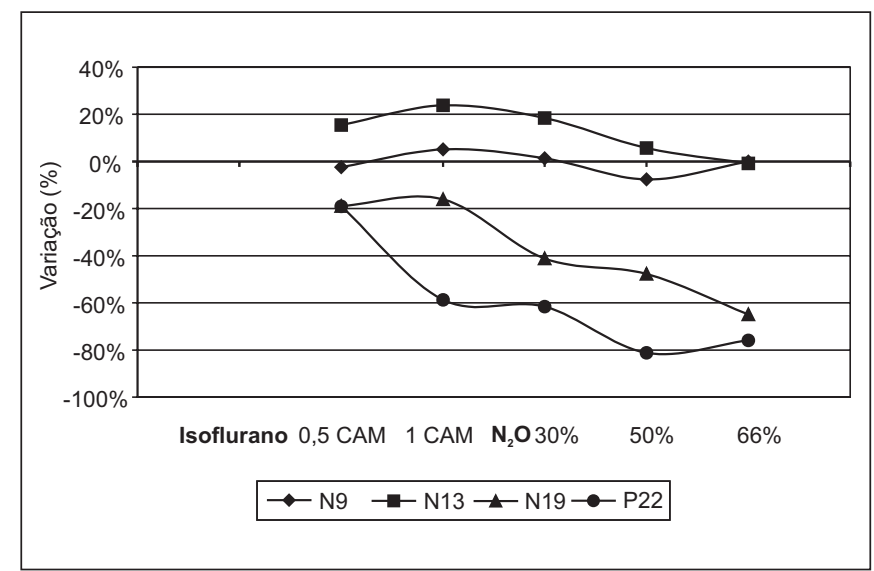

Figura 7 - Variação Percentual da Amplitude das Ondas PESS com Işoflurano Administrado como Agente Único e Associado ao Óxido Nitroso

( $p=0,15$ comparando 1 CAM a 1 CAM associado a $30 \%$ de óxido nitroso)

( $p<0,06$ comparando 1 CAM a 1 CAM associado a $50 \%$ de óxido nitroso)

( $p<0,001$ comparando 1 CAM a 1 CAM associado a $66 \%$ de óxido nitroso)

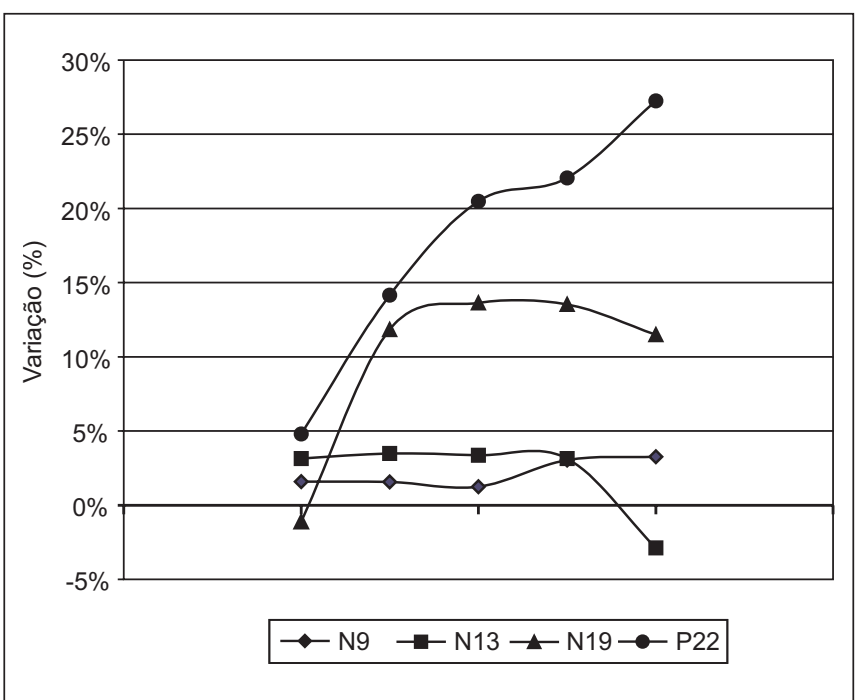

Figura 8 - Variação Percentual da Latência das Ondas do PESS com Isoflurano Administrado como Agente Único e Associado ao Óxido Nitroso

( $p=0,043$ com $F A 30 \%$ de óxido nitroso; $p=0,013$ com FA $50 \%$ de óxido nitroso e $p=0,066$ com FAde $66 \%$ de óxido nitroso) 


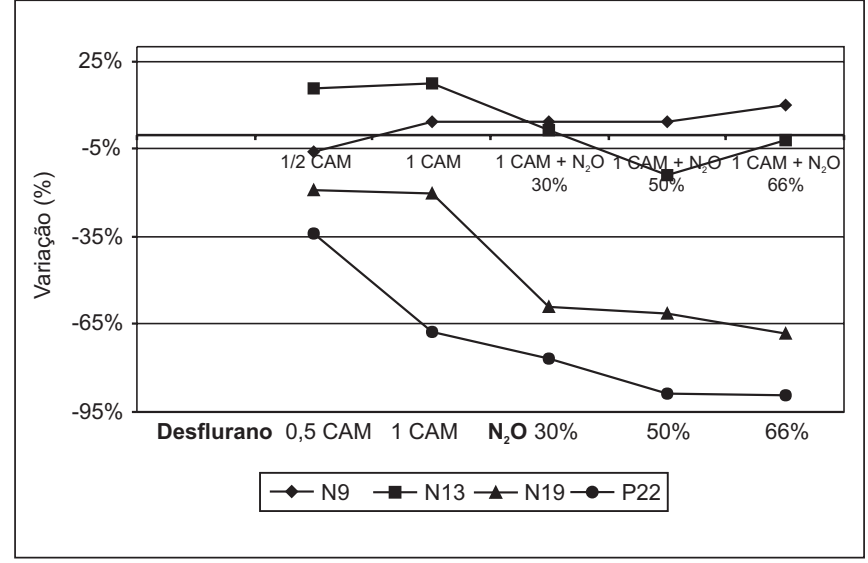

Figura 9 - Variação Percentual da Amplitude das Ondas PESS com Desflurano Administrado como Agente Único e Associado ao Óxido Nitroso

$(p=0,03$ com $F A 30 \%$ de óxido nitroso; $p=0,05$ com FA $50 \%$ de óxido nitroso e $p=0,04$ com FA $66 \%$ de óxido nitroso)

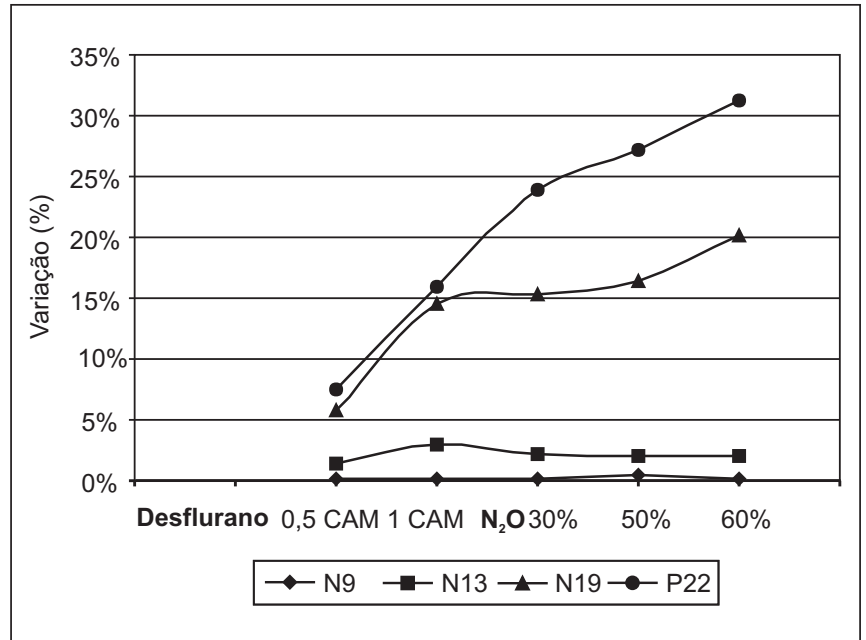

Figura 10 - Variação Percentual da Latência das Ondas PESS com Desflurano Administrado como Agente Único e Associado ao Óxido Nitroso

( $p=0,005$ com FA $30 \% ; p=0,007$ com FA $50 \%$ e $p=0,009$ com FA $66 \%$ de óxido nitroso)

\section{DISCUSSÃO}

Os resultados encontrados nas variáveis fisiológicas estudadas estão de acordo com achados de outros autores, evidenciando a preservação estável das funções cardiorrespiratórias quando o óxido nitroso foi utilizado como agente único ou associado a outros agentes em concentrações alveolares que foram de 30,50 e $66 \%$. Nas citadas concentrações, o óxido nitroso não desenvolve ação nas estruturas nervosas que possam inibir ou liberar determinados neurotransmissores autonômicos capazes de promover alterações cardiovasculares e tampouco inibir o automatismo respiratório causando hipoventilação alveolar ${ }^{12,14,15}$.
As pequenas alterações encontradas no valor do BIS com a utilização de óxido nitroso indicam que este tem uma ação hipnótica muito leve.

Neste estudo o BIS tinha o valor basal (média) de 97,12 e foi reduzido para 74,00 quando o óxido nitroso foi administrado a $30 \%$. Este valor foi praticamente mantido $(76,64$ e 77,64$)$ quando foi administrado 50 e $66 \%$ de óxido nitroso respectivamente.

Há relato na literatura de que valores do BIS entre 60 e 80 foram associados com aumento da probabilidade de consciência ${ }^{16}$. Portanto, apesar de ter havido uma diminuição, os valores atingidos em todas as concentrações estudadas não indicam um pleno estado de hipnose. A diminuição que ocorreu no início, com a FA de óxido nitroso a 30\%, poderia estar relacionada a um efeito residual do propofol venoso utilizado na indução anestésica. No entanto, a redução do valor do BIS após uma dose em bolus de propofol é bem maior, indicando hipnose plena, e demora apenas 8 minutos para que o valor volte a se elevar para mais de 60, e logo a seguir havero retorno da consciência ${ }^{17}$. Neste estudo, após o início do efeito do propofol, havia um intervalo de 30 minutos durante o qual nenhum anestésico era utilizado. O valor do BIS não retornava para o valor basal, mas chegava aproximadamente a 80. Este valor está obviamente mais próximo do estado de alerta do que do estado de hipnose. Estudos mostram que pacientes que receberam apenas óxido nitroso a 10, 20,30, 40 e $50 \%$ não apresentaram nenhuma mudança no valor do $\mathrm{BIS}^{18}$. Um outro estudo mostrou que, mesmo com óxido nitroso a $70 \%$, também não houve nenhuma mudança significativa no BIS que viesse a caracterizar hipnose ${ }^{19}$.

$O$ isoflurano e o desflurano reduzem o valor do BIS e esta redução é dose-dependente. Este resultado mostra que estes agentes têm ação hipnótica bem pronunciada, diferente do óxido nitroso. Estes mesmos resultados, com drogas como propofol, midazolam e isoflurano, já foram demonstrados por outro autor ${ }^{20}$. Há descrição de aumento no valor do BIS com uso de concentração elevada de isoflurano ${ }^{21}$. Neste estudo não foi encontrado aumento do valor do BIS quando a concentração do isoflurano foi aumentada, como relatado por aquele autor, embora não tenha sido ultrapassado a dose de 1 CAM. Talvez esta concentração limitada justifique a diferença de resultados. Com relação ao desflurano, não há estudos na literatura em concentrações semelhantes às utilizadas neste trabalho.

A associação do óxido nitroso ao isoflurano e desflurano a 1 CAM promoveu uma pequena diminuição do BIS, não ultrapassando $10 \%$, em relação ao valor obtido com os agentes administrados isoladamente. A diminuição foi um pouco maior nos pacientes que receberam desflurano. Estes resultados concordam com um estudo anterior no qual o autor não encontrou nenhuma diferença entre o valor do BIS quando o óxido nitroso foi utilizado como agente único (70\%) ou associado ao isoflurano, apesar de ter ocorrido perda da consciência ${ }^{19}$. Outro autor revelou que a administração de $50 \%$ de óxido nitroso como agente único produz pouca sedação e nenhuma mudança no BIS. No entanto, foi observado que havia ativação de certas áreas no EEG não 
captadas pelo BIS, indicando que o óxido nitroso tem efeitos excitatórios e inibitórios no SNC ${ }^{18}$.

Há relatos de trabalhos mostrando ações diferentes do óxido nitroso sobre o SNC, diferindo inclusive dos resultados encontrados neste estudo. É descrito que o óxido nitroso antagoniza os efeitos depressivos do isoflurano no EEG ${ }^{9,22}$. Existe relato de que o valor do BIS foi mais alto quando o isoflurano foi usado em associação com óxido nitroso do que como agente único ${ }^{23}$. Outro estudo mostrou que a adição de óxido nitroso ao propofol aumentou o BIS que estava reduzido, ainda que os pacientes não tenham respondido ao comando verbal ${ }^{24}$, certamente devido ao estado de amnésia. Na verdade, os estudos do efeito do óxido nitroso sobre o BIS são conflitantes, refletindo possivelmente uma inadequação do algorritmo usado no cálculo do BIS proveniente do EEG. Estes achados questionam a eficiência do BIS como monitor da profundidade anestésica em pacientes recebendo óxido nitroso como agente único ou associado a outro agente anestésico.

O óxido nitroso não promoveu ação inibitória sobre as estruturas nervosas periféricas, como o plexo braquial, e tampouco sobre a medula espinhal nas concentrações em que foi utilizado. É possível que exista alguma ação nestas estruturas, especialmente a medula, e que devido à pequena magnitude, não tenha sido captada pelas ondas N9 e N13 do PESS. Deve ser considerado que a concentração mais elevada utilizada correspondia a aproximadamente 0,6 CAM deste agente. Geralmente as concentrações que promovem inibição, mesmo leve, sobre a medula espinhal com outros agentes, são geralmente múltiplos da CAM (1,3 a 1,5 CAM). O isoflurano e o desflurano também não alteraram os potenciais periféricos e medular. A ação destes agentes na condução do estímulo através dos nervos periféricos e na medula parece ser muito pequena, não sendo suficiente para alterar os potenciais gerados no plexo braquial e ao nível de C2. Estes achados são semelhantes aos de outro estudo em que o autor não encontrou alterações dos potenciais subcorticais promovidos pelo óxido nitroso ${ }^{9}$. Em outro estudo, também sobre a ação do óxido nitroso no PESS, o autor descreveu alteração não significativa na amplitude da onda N13 com óxido nitroso como agente único e também associado ao isoflurano. No entanto, este mesmo autor concorda que a anestesia tem um efeito mínimo nos componentes cervical (N13) e do plexo braquial (N9), e que os resultados por ele encontrados discordam dos descritos em observações anteriores ${ }^{25}$. É postulado que as respostas mediadas nas ondas N9 e N13 são mais dependentes da condução axonal do que da transmissão sináptica, sendo desta forma estes potenciais menos influenciados pela anestesia geral ${ }^{26}$.

O óxido nitroso tem ação inibitória sobre estímulos ascendentes direcionados ao cérebro. Esta inibição pode ser observada logo na entrada dos estímulos na estrutura cerebral, evocados na onda N19, e também depois no córtex pela inibição da onda P22. Houve redução significativa na amplitude destas ondas.

A onda N19 não apresentou alterações de latência. A redução na amplitude ocorreu em todas as concentrações estudadas, sendo máxima quando a FA de óxido nitroso era de
$50 \%$. Na onda P22 também não houve alteração na latência e a amplitude reduziu $38,52 \%$ com o óxido nitroso administrado a $50 \%$. Sendo elevada a $66 \%$, a redução na amplitude passou a ser de $46,48 \%$, havendo um declínio de apenas $12,95 \%$ sobre o valor anterior (FA50). É importante ressaltar que a redução na amplitude não foi acentuada com o aumento da FA de 50 para $66 \%$ na onda N19. É descrito por outros autores a redução da amplitude promovida pelo óxido nitroso, sem promover alteração na latência das ondas corticais $10,25,27$. No entanto, nenhum destes estudos mostrou reduções percentuais da amplitude muito próximas na onda N19 em concentrações diferentes, como foi encontrado no presente estudo com FA de óxido nitroso a 50 e $66 \%$, e também valores de amplitude bem próximos nas mesmas concentrações na onda P22. Provavelmente isto não foi demonstrado em outros estudos porque o método não favoreceu os resultados, ou seja, o autor estudou apenas uma concentração, ou então estudou a FA de $50 \%$ como concentração máxima de óxido nitroso.

Há relato na literatura de que a ação depressora do óxido nitroso na amplitude das ondas corticais, avaliada pelo PESS, é mais pronunciada do que a do isoflurano ${ }^{9,25}$. No presente estudo a redução na amplitude da onda cortical P22 foi maior com o óxido nitroso na FA de 30,50 e $66 \%$ de $\mathrm{N}_{2} \mathrm{O}$ do que com 0,5 CAM de isoflurano. $\mathrm{Na}$ onda N19, a depressão causada pela FAde 50 e $66 \%$ foi maior do que a encontrada com 0,5 e 1 CAM de isoflurano. Isto sugere que o óxido nitroso, em doses equipotentes da CAM, tem efeito depressor no córtex primário maior do que o isoflurano. Isto pode refletir o bom efeito analgésico e amnésico do óxido nitroso, em contraste ao seu fraco efeito hipnótico. A estimulação da produção de opióides endógenos a nível subcortical e o efeito depressor direto na área cortical foram propostos como mecanismos responsáveis pelo seu efeito analgésico ${ }^{28}$. Com relação ao desflurano, pode-se observar que a redução na amplitude das ondas cerebrais que promove com 0,5 CAM é menor do que a promovida pelo óxido nitroso na FA de 50 e $66 \%$. Não foi encontrado nenhum estudo na literatura que comparasse a ação do óxido nitroso ao desflurano, mas pelos resultados encontrados no presente estudo, em doses equipotentes da CAM, a ação depressora do óxido nitroso no córtex é maior do que 0,5 CAM de desflurano. Tal fato também ressalta os mecanismos responsáveis pelo bom efeito analgésico e amnésico desse agente.

Aassociação do óxido nitroso ao isoflurano e desflurano produziu alterações importantes, sobretudo na amplitude das ondas N19 e P22, com pequenas alterações na latência desta última. Estes resultados estão de acordo com os de outros autores mostrando que a adição de $60 \%$ de óxido nitroso causa redução significativa na amplitude das ondas corticais ${ }^{28-31}$. Nesse estudo a adição de óxido nitroso ao isoflurano reduziu ainda mais a amplitude das ondas cerebrais. Na onda N19 as reduções foram mais acentuadas à medida que maiores FA de óxido nitroso foram administradas. $\mathrm{Na}$ onda $\mathrm{P} 22$ a redução máxima foi na FA de 50\%. Haveria então uma concentração limite a partir da qual o aumento da FA de óxido nitroso não acentuaria a redução da amplitude promovida por 1 CAM 
de isoflurano nesta onda? Com o desflurano, as ondas $\mathrm{N} 19 \mathrm{e}$ P22 apresentaram maior declínio no intervalo entre o valor basal e aquele com FA de óxido nitroso a $50 \%$. A partir desta FA, o declínio passa a ser insignificante (Figuras 9 e 10). $O$ aumento da FA não resulta em depressão proporcional das ondas cerebrais, o que provavelmente pode ser atribuída à existência de "efeito teto". Certamente outros estudos necessitam ser desenvolvidos neste sentido.

Neste estudo foi encontrado aumento maior na latência da onda P22 quando o óxido nitroso foi associado a 1 CAM de isoflurano e de desflurano. Já foi descrito que o óxido nitroso em FA de $60 \%$ associado a 0,6 CAM de isoflurano produz um leve aumento adicional na latência da onda N19, além daquele produzido apenas por 0,6 CAM de isoflurano. $O$ autor encontrou aumento de apenas 1 milissegundo na latência, que não foi clinicamente importante ${ }^{25}$. A latência é raramente usada como critério de alteração durante monitorização per-operatória por causa da sua baixa sensibilidade ${ }^{32}$. No entanto, verificamos neste estudo que a latência da onda P22 aumentou ainda mais com a "adição" de óxido nitroso em todas as concentrações estudadas. Estes achados concordam com estudo anterior no qual os autores descreveram aumento ainda maior na latência das ondas corticais, promovido pela "adição" de óxido nitroso (FAde 60\%), ao sevoflurano ${ }^{33}$. Há relatos na literatura de alterações no PESS promovidas por variações de $\mathrm{P}_{\mathrm{ET}} \mathrm{CO}_{2}{ }^{34}$, hipotermia ${ }^{35}$, hipertermia ${ }^{36}$, e estímulo cirúrgico ${ }^{27}$. Neste estudo, os pacientes estavam sob anestesia peridural, não tendo havido variações importantes de $\mathrm{P}_{\mathrm{ET}} \mathrm{CO}_{2}$ e de temperatura. Portanto, todas as alterações encontradas no BIS e PESS podem ser atribuídas em especial ao óxido nitroso, assim como em determinado momento ao isoflurano e ao desflurano.

Ao mesmo tempo em que o óxido nitroso promove redução definida na amplitude dos potenciais corticais, ele altera pouco o BIS, podendo até eventualmente promover elevação em seu valor. Provavelmente em seu mecanismo de ação exista um aumento da inibição córtico-talâmica e diminuição da excitação ${ }^{37}$. No entanto, já foi relatada uma ativação promovida pelo óxido nitroso em certas áreas do córtex cerebral captadas pelo EEG ${ }^{38}$, que pode ser atribuída a um efeito estimulante direto no córtex cerebral gerando surtos espontâneos, ao mesmo tempo em que há redução na transmissão de informações sensoriais para o córtex cerebral pelo bloqueio do núcleo talâmico. Isto resulta na redução da amplitude das ondas cerebrais. Também tem sido observado que a ativação dos centros reticulares cerebrais promovida pelo óxido nitroso pode ser responsável pela ativação de áreas cerebrais captadas pelo EEG ${ }^{39}$.

Em conclusão, foi demonstrado que o óxido nitroso altera pouco a recepção de estímulos espontâneos que chegam ao córtex cerebral e são captados pelo eletroencefalograma. Altera levemente os potenciais medulares e periféricos quando utilizado como agente único e associado ao isoflurano e desflurano. Reduz significantemente a amplitude dos potenciais corticais como agente único e associado ao isoflurano e desflurano, o que reflete o seu forte efeito analgésico e seu fraco efeito hipnótico.

\section{AGRADECIMENTOS}

Os autores agradecem à equipe de neurofisiologia clínica: os médicos Alexandre Cardoso Almeida e Maria Dorvalina Silva e os técnicos Josias Francisco de Macedo, Joselda Maria Vieira Lobo, Raimunda Nonata Melo Rosendo e Renata Rodrigues dos Santos do Hospital Sarah-Brasília e em especial ao estatístico Márcio Correa de Mello, pelo apoio à realização deste estudo.

\section{Nitrous Oxide Action on the Central Nervous System. Electrophysiological Study as a Sole Agent or a Coadjuvant}

\author{
Verônica Vieira da Costa, M.D., Renato Ângelo Saraiva, \\ TSA, M.D.
}

\section{INTRODUCTION}

Nitrous oxide is a gaseous anesthetic agent of low potency. Its minimum alveolar concentration (MAC) is $104 \%( \pm 10)$, which corresponds to a partial pressure of $805 \mathrm{mmHg}$ (at sea level), only experimentally obtained with a hyperbaric chamber ${ }^{1}$ or by calculated estimates.

It is used in non-hypoxic concentrations, never above $70 \%$. Nitrous oxide has moderate analgesic properties, weak amnesic action, minor immobilizing power and very mild hypnotic effect ${ }^{2-4}$. Hence, its indications as sole anesthetic agent are very limited, being used mostly a coadjuvant of more potent inhalational anesthetic to decrease their doses and, as a consequence, their side-effects.

Nitrous oxide low blood and tissue solubility (blood/gas coefficient of 0.47 and brain/blood coefficient of 1.1$)^{5}$ provides it with very special and desirable pharmacokinetic properties, especially as a coadjuvant, since its uptake and distribution are very fast, as well as its excretion. Its pharmacodynamic profile indicates very mild side effects, with emphasis to minimum cardiovascular and respiratory effects. In addition, it is a less soluble gas with almost no metabolization. It is widely used for being very well tolerated ${ }^{6,7}$.

With a MAC very close to $100 \%$, when used as a coadjuvant drug, it should theoretically decrease main agent's MAC in a proportion equivalent to its own the concentration being administered. For example, since halothane MAC is about $0.76 \%$, the addition of nitrous oxide to the inhalational mixture in a $50 \%$ concentration would decrease this MAC to $0.38 \%$.

According to the literature, this proportion of reduction of volatile anesthetic's MAC is observed for same agents, but not to all ${ }^{8}$. In addition, some electrophysiological studies have shown a linear relationship of this additive effect on certain parts of the central nervous system (CNS) ${ }^{9}$, but not on all CNS structures ${ }^{10}$. 
Nitrous oxide pharmacological characteristics are widely studied. It is not considered a complete anesthetic agent, although exhibiting analgesic and amnesic properties. It is not hypnotic and may cause a hilarious effect. It may cause excitation by acting as a depressor on CNS areas with inhibiting functions, releasing other areas with stimulating functions and triggering a typically extrapiramidal reaction. Nitrous oxide's action mechanism on CNS structures is not thoroughly known. However, a lot is already known through evidences found in experimental studies and clinical trials ${ }^{11,12}$.

Nitrous oxide produces very typical clinical states, different from other inhalational anesthetics. It is possible that an electrophysiological evaluation may reveal results showing how different are its action's on different CNS structures. The challenge of observing functional neurological changes during nitrous oxide administration is a very interesting motivation. So, the aim of this study was to objectively evaluate, thru specific monitoring, the action of such drug on CNS structures, both as sole agent or as a coadjuvant, correlating observed changes to its pharmacological effects.

\section{METHODS}

After the Hospital's Ethical Committee approval, 25 patients of both genders, aged 6 to 25 years, physical status ASA I and II, undergoing lower limb orthopedic or corrective plastic surgeries were included in this study. Patients were premedicated with $0.8 \mathrm{mg} \cdot \mathrm{kg}^{-1}$ oral midazolam 30 minutes before surgery, with a maximum dose of $15 \mathrm{mg}$. Routine physiological and electroneurophysiological monitoring were installed in the anesthetic induction room.

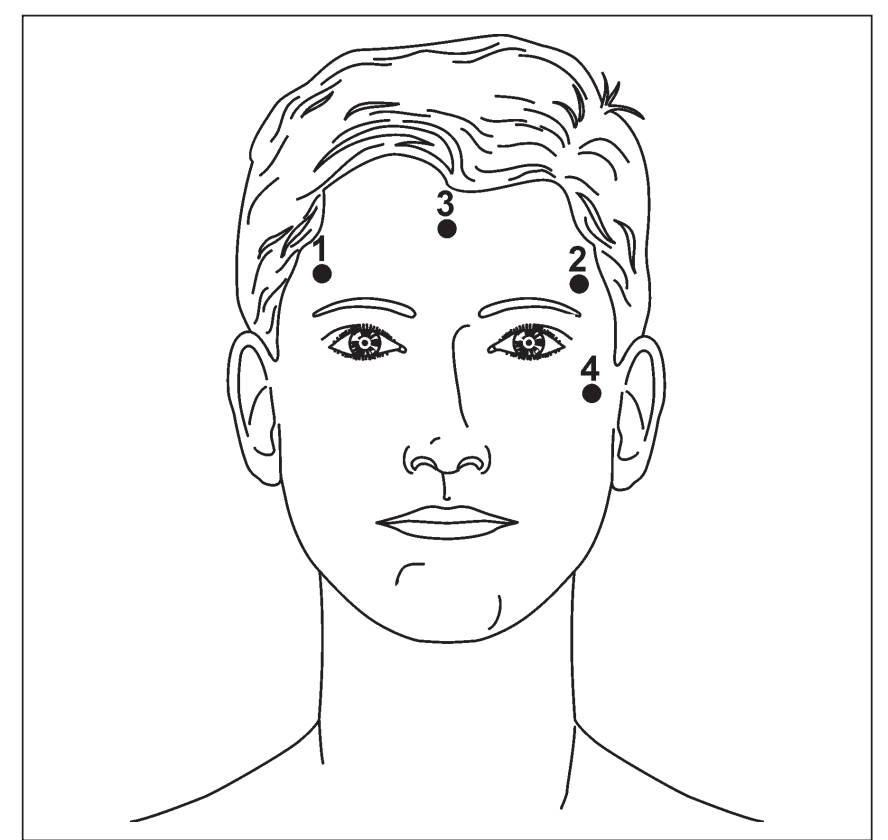

Figure 1 - BIS Electrodes Placement According to the 10-20 International System
Electroencephalogram with BIS was used to monitor the actions on brain cortex, with Zipprep-type electrodes (Aspect Medical System) placed on the frontal region; one electrode on each side and a third reference electrode between them. Two channels, were established (Fp1 and Fp2) plus a referential channel (Fpz), according to the International System 10-20 of the International Society of Clinical Neurophysiology (Figure 1). Fp1-Fpz was analyzed in channel 1, Fp2-Fpz in channel 2 and the "ground" placed on the pre-auricular region. BIS and both BIS channel waves were recorded from successive 2 seconds periods ${ }^{13}$ and updated at every 5 seconds by a monitor A 1000 (Aspects Medical Systems, Natick, MA). Selected frequency bandwidth was set between 0.5 and $30 \mathrm{Hertz}(\mathrm{Hz})$. Impedance was checked before every reading and remained always below 800 ohms $(\Omega)$. The monitor also featured an automatic artifact detection and rejection system. BIS baseline value was obtained before anesthetic induction.

Anesthesia was induced with $3 \mathrm{mg} \cdot \mathrm{kg}^{-1}$ propofol and 0.1 $\mathrm{mg} . \mathrm{kg}^{-1}$ vecuronium, followed by tracheal intubation and mechanical ventilation. All patients received epidural anesthesia between the third and fourth lumbar vertebra with bupivacaine in concentration and volume depending on patients weight, but never exceeding $3 \mathrm{mg} \cdot \mathrm{kg}^{-1}$. The purpose of that was to avoid the interference of other stimuli on the monitor. Somatosensory evoked potential (SEP) was recorded 30 minutes after propofol administration, being this first record considered the baseline value, thus avoiding artifacts generated by muscle shivering due to low operating room temperatures. SEP was monitored by a Dantek-Keypoint release 3.04 monitor (Denmark) after right median nerve stimulation with a bar-type electrode, with a cathode-anode distance of $3.5 \mathrm{~cm}$. Records were obtained through surface electrodes on the following sites: Erb point, located on the right supraclavicular fossa (wave N9); over the spinous process of the second cervical vertebra (N13); over the skull, close to parietal lobe sensory cortex, contralateral to the stimulated limb (waves N19 and P22), according to the International System 10-20. The reference electrode (Fz) was placed in the top of the middle frontal region, and the "ground" electrode was placed on the right shoulder. Impedance was always kept below 5 kohms $(\Omega)$. Stimulation intensity was adjusted until motor response threshold was reached, characterized by the Thumb Twith. For each SEP reading, 20 constant current impulses were repeated for 0.2 miliseconds (ms), with a discharge frequency of $5.1 \mathrm{~Hz}$. Filtration band was adjusted between $20 \mathrm{~Hz}$ (low) and $3000 \mathrm{~Hz}$ (high). Promediations (tracings) were duplicated and both record series were compared to evaluate their reproducibility.

Waves N9 (brachial plexus), N13 (cervical spinal cord), N19 (thalamus-cortical) and P22 (cortical) were simultaneously evaluated. Onset time was recorded in milliseconds ( $\mathrm{ms}$ ) and amplitude in microvolts ( $\mu \mathrm{v})$.

Regular monitoring consisted of ECG, non-invasive blood pressure (BP), hemoglobin oxygen peripheral saturation $\left(\mathrm{SpO}_{2}\right)$, end expired $\mathrm{CO}_{2}$ partial pressure $\left(\mathrm{P}_{\mathrm{ET}} \mathrm{CO}_{2}\right)$ and nasopharyngeal temperature, as well as inspired and end expired 
concentration (alveolar) of inhaled agents through an anesthetic gas analyzer. After baseline SEP recording, nitrous oxide was introduced until an alveolar fractional concentration (AF) of $30 \%$ was reached. After 5 minutes, a new SEP was recorded. While SEP was being registered, BIS values on the screen were recorded as well with mean values being calculated. At the same time, the other physiological variables values were also registered. Then, nitrous oxide concentration was increased to $50 \%$ and $66 \%$, following the same recording procedures. Next, nitrous oxide was withdrawn and isoflurane or desflurane were randomly introduced until an AF of $0.5 \mathrm{MAC}$. After 10 minutes BIS, SEP and other variables were once again recorded, the same being repeated after bringing AF to $1 \mathrm{MAC}$. With the patients at $1 \mathrm{MAC}$ of their respective agents, nitrous oxide was reintroduced in the same concentrations $(30 \%, 50 \%$ and $66 \%)$ and the same procedures were performed again, in the previous sequence and time intervals.

Student's $t$ and Chi-square tests were used for statistical analysis of demographics. Bonferroni's method was used to compare cardiovascular, respiratory and temperature variables between different nitrous oxide and other agents concentrations. Exploratory data analysis (mean and standard deviation) was used to evaluate the significance of neurophysiological changes, followed by the method of repetitive measures (modified Fisher's test), considering statistically significant $p \leq 0.05$.

\section{RESULTS}

Twenty-five patients were given initially nitrous oxide as sole agent, which was then temporarily withdrawn. After that, 13 patients were given isoflurane and 12 desflurane, according to the above mentioned sequence. There were no differences in gender, age, physical status and weight between the group receiving nitrous oxide and isoflurane and the group receiving nitrous oxide and desflurane (Table I).

There were no significant changes in physiological variables during nitrous oxide administration in different concentrations. Stability was maintained with sevoflurane and desflurane, with a mild trend to decrease those values, but with no statistical or clinical significance. Mean $\mathrm{P}_{\mathrm{ET}} \mathrm{CO}_{2}$ values varied from 39 to $33 \mathrm{mmHg}$, reaching lower values in the isoflurane group, but also with no statistical significance. There were no significant temperature changes as well (Tables II and III).

Table I - Demographics Data

\begin{tabular}{|c|c|c|c|c|c|}
\hline & \multicolumn{2}{|c|}{$\mathrm{N}_{2} \mathrm{O}+$ Desflurane } & \multicolumn{2}{|c|}{$\mathrm{N}_{2} \mathrm{O}+$ Isoflurane } & $p$ value \\
\hline Age (years)* & \multicolumn{2}{|c|}{$12.50 \pm 2.355$} & \multicolumn{2}{|c|}{$14.00 \pm 2.9786$} & $0.443^{* *}$ \\
\hline Weight $(\mathrm{kg})^{*}$ & \multicolumn{2}{|c|}{$38.58 \pm 8.326$} & \multicolumn{2}{|c|}{$47.04 \pm 10.232$} & $0.213^{* *}$ \\
\hline \multirow[t]{2}{*}{ Gender } & M & $\mathrm{F}$ & M & $\mathrm{F}$ & \multirow{2}{*}{$0.695^{* * *}$} \\
\hline & $6 / 12$ & $6 / 12$ & $8 / 13$ & $5 / 13$ & \\
\hline \multirow[t]{2}{*}{ Physical status (ASA) } & I & II & 1 & II & \multirow[b]{2}{*}{-} \\
\hline & $11 / 12$ & $1 / 12$ & $12 / 13$ & $1 / 13$ & \\
\hline
\end{tabular}

* Values expressed in Mean $\pm 2 \mathrm{SD}$; ${ }^{* *}$ Student's $t$ test; *** Chi-square test

Table II - Physiological Variables Analyzed during Nitrous Oxide Administration as a Sole Agent

\begin{tabular}{lcccrr}
\hline $\mathrm{N}_{2} \mathrm{O}$ Concentration & Mean Blood Pressure & Heart Rate & Temperature & $\mathrm{P}_{\mathrm{ET}} \mathrm{CO}_{2}$ & SpO \\
\hline Baseline & $91.35 \pm 11.86$ & $89.68 \pm 22.52$ & $36.22 \pm 1.14$ & - \\
$30 \% \mathrm{~N}_{2} \mathrm{O}$ & $98.76 \pm 17.61$ & $107.12 \pm 20.57$ & $36.11 \pm 1.04$ & $39.88 \pm 5.87$ \\
$50 \% \mathrm{~N}_{2} \mathrm{O}$ & $101.73 \pm 14.46$ & $103.16 \pm 22.72$ & $36.08 \pm 1.15$ & $99.96 \pm 0.28$ & $39.64 \pm 4.73$ \\
$66 \% \mathrm{~N}_{2} \mathrm{O}$ & $96.11 \pm 15.63$ & $96.92 \pm 23.70$ & $36.01 \pm 1.10$ & $38.84 \pm 4.95$ \\
\hline
\end{tabular}

* Values expressed in Mean $\pm \mathrm{SD}$

Table III - Physiological Variables Analyzed during Nitrous Oxide Administration Associated to Isoflurane and Desflurane

\begin{tabular}{|c|c|c|c|c|c|c|}
\hline $\mathrm{N}_{2} \mathrm{O}$ Concentration & Anesthetics & Mean Blood Pressure & Heart Rate & Temperature & $\mathrm{P}_{\mathrm{ET}} \mathrm{CO}_{2}$ & $\mathrm{SpO}_{2}$ \\
\hline \multirow[t]{2}{*}{$1 \mathrm{MAC}$} & Isoflurane & $74.31 \pm 15.63$ & $80.92 \pm 22.6$ & $35.53 \pm 1.28$ & $34.08 \pm 3.28$ & $99.62 \pm 0.65$ \\
\hline & Desflurane & $75.67 \pm 17.87$ & $89.25 \pm 25.11$ & $35.96 \pm 0.74$ & $36.00 \pm 2.22$ & $99.50 \pm 0.52$ \\
\hline & Desflurane & $68.33 \pm 8.89$ & $84.00 \pm 21.59$ & $35.91 \pm 0.67$ & $38.00 \pm 2.37$ & $99.33 \pm 0.49$ \\
\hline \multirow[t]{2}{*}{$1 \mathrm{MAC}+50 \% \mathrm{~N}_{2} \mathrm{O}$} & Isoflurane & $65.23 \pm 7.11$ & $75.62 \pm 19.89$ & $35.21 \pm 1.32$ & $34.46 \pm 2.76$ & $99.46 \pm 0.78$ \\
\hline & Desflurane & $69.13 \pm 8.24$ & $83.00 \pm 21.15$ & $35.88 \pm 0.68$ & $38.25 \pm 2.77$ & $99.00 \pm 0.6$ \\
\hline \multirow[t]{2}{*}{$1 \mathrm{MAC}+66 \% \mathrm{~N}_{2} \mathrm{O}$} & Isoflurane & $64.54 \pm 7.43$ & $74.08 \pm 19.96$ & $35.07 \pm 1.23$ & $33.85 \pm 2.88$ & $99.00 \pm 0.91$ \\
\hline & Desflurane & $65.58 \pm 7.54$ & $83.75 \pm 19.92$ & $35.72 \pm 0.6$ & $37.08 \pm 4.83$ & $98.67 \pm 1.07$ \\
\hline
\end{tabular}

* Values expressed in Mean \pm SD 
EEG-BIS Monitoring of Nitrous Oxide, Isoflurane and Desflurane Effects on the Brain Cortex

BIS value decreased $23.8 \%$ when $30 \%$ nitrous oxide was used as a sole agent, reaching statistical significance $(p<0.001)$. As concentration increased to $50 \%$ and $66 \%$, BIS values were kept stable, with only a slight trend to increase (Figure 2). After nitrous oxide withdrawal (AF close to zero) and 0.5 MAC isoflurane achieved, there was a $42.6 \%$ BIS decrease as compared to baseline, with statistical significance ( $p<$ 0.001 ). When the concentration was increased to $1 \mathrm{MAC}, \mathrm{BIS}$ had a further drop to $66.17 \%$ of baseline $(p<0.001)$.

With 0.5 MAC desflurane, there was a $46.9 \%$ BIS reduction ( $p$ $<0.001$ ), and a $67.93 \%$ decrease when concentration was increased to 1 MAC $(p<0.001)$.

With $1 \mathrm{MAC}$ isoflurane and nitrous oxide readministration in increasing concentrations ( $30 \%, 50 \%$ and $66 \%)$, there was a mild BIS decrease of $3.26 \%, 5.59 \%$ and $10.96 \%$, respectively. At the same time, desflurane group showed a BIS reduction of $9.95 \%, 16.67 \%$ and $24.73 \%$, respectively. BIS decrease was higher in patients where nitrous oxide was associated to desflurane compared to those where nitrous oxide was associated to isoflurane. Prior to nitrous oxide reintroduction, isoflurane and desflurane produced a sudden and squared wave form BIS decrease (Figure 3). When nitrous oxide was associated to those potent agents, BIS reduction was smoother, with a trend to stabilization from $50 \%$ concentration on.

Nitrous Oxide, Isoflurane and Desflurane Effects on Peripheral Nerve, Spinal Cord and Brain, evaluated by Peripheral Nerve Somatosensory Evoked Potential - SEP

Action on peripheral nerve structure (brachial plexus) -SEP Wave N9:

Nitrous oxide as sole agent did not change amplitude and onset time with $30 \%, 50 \%$ and $66 \%$ alveolar fractions (AF).

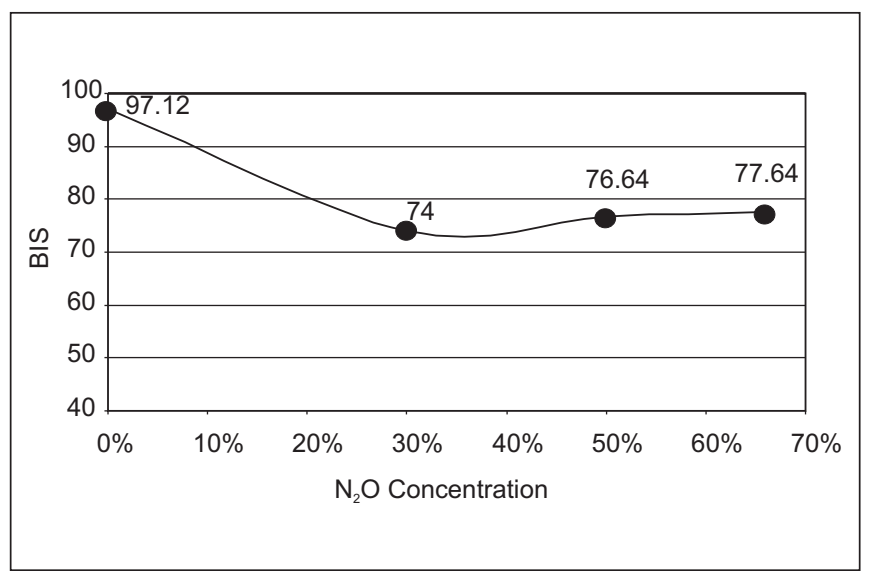

Figure 2 - BIS Mean Value Variation with Nitrous Oxide $\left(\mathrm{N}_{2} \mathrm{O}\right)$ as a Sole Agent

$(p<0.001$ between baseline value and at $30 \%$ nitrous oxide AF)

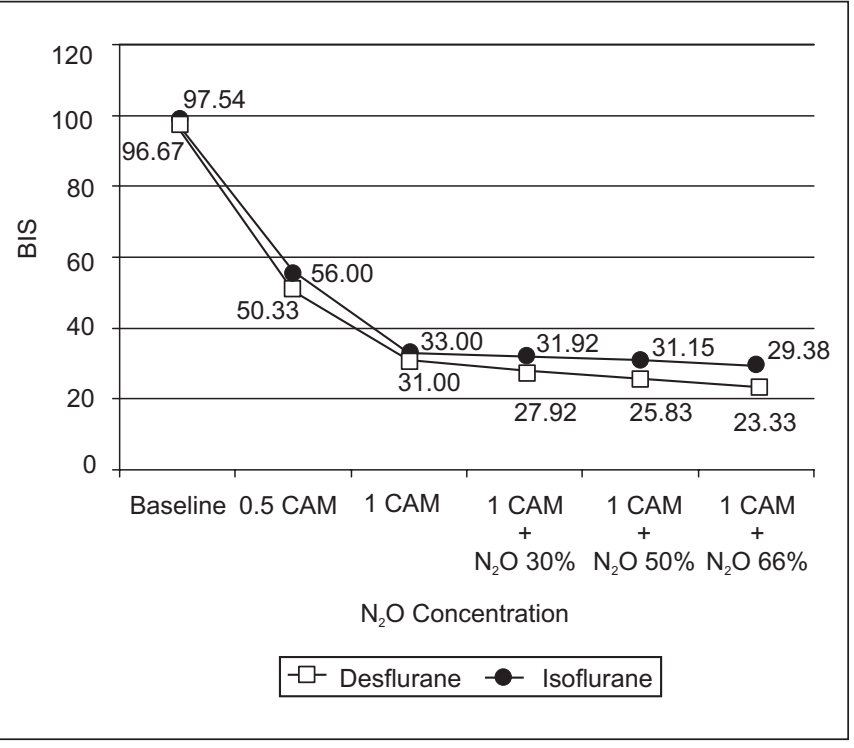

Figure 3 - BIS Mean Value Variation with Nitrous Oxide Associated to Isoflurane and Desflurane

$(p<0.001$ between baseline value and 0.5 MAC AF, and between baseline value and $1 \mathrm{MAC} A F$ )

Isoflurane and desflurane also did not affect amplitude and onset time when administered in AF of 0.5 and 1 MAC.

Similarly, $30 \%, 50 \%$ and $66 \%$ nitrous oxide AF associated to $1 \mathrm{MAC}$ isoflurane or desflurane had no influence on amplitude and onset time of wave N9.

Action on Spinal Cord Structure - SEP Wave N13:

There were no significant amplitude and onset time changes with $30 \%, 50 \%$ and $66 \%$ nitrous oxide, as well as with isoflurane or desflurane at 0.5 and 1 MAC.

Action on Thalamus-brain Structure - Wave N19:

Nitrous oxide decreased its amplitude in $10.76 \%$ at $30 \% \mathrm{AF}$ compared to baseline. With $50 \% \mathrm{AF}$, the decrease was $29.57 \%$, and $28.09 \%$ with $66 \%$ AF $(p<0.001)$. There was no change in onset time with those concentrations (Figures 4 and 5).

Isoflurane promoted an $18.93 \%$ amplitude decrease at 0.5 MAC ( $p=0.303)$ and $16.18 \%$ at 1 MAC $(p=0.42)$ compared to baseline. Onset time had a $3.72 \%$ increase at $0.5 \mathrm{MAC}$ and $11.84 \%$ at 1 MAC. Such changes, however, were not statistically significant.

Desflurane reduced amplitude in $19.21 \%$ at 0.5 MAC ( $p=$ $0.37)$ and $20 \%$ at $1 \mathrm{MAC}(\mathrm{p}=0.42)$ compared to baseline. Onset time increased $5.90 \%$ at $0.5 \mathrm{MAC}$ and $14.60 \%$ at $1 \mathrm{MAC}$ compared to baseline. Those changes were also not statistically significant.

The association of $1 \mathrm{MAC}$ isoflurane to $30 \%, 50 \%$ and $66 \%$ nitrous oxide decreased amplitude even further to $29.92 \%(p=$ $0.137), 37.64 \%(p=0.067)$ and $58.20 \%(p=0.014)$, respectively. Onset time showed no further increase.

One MAC desflurane associated to $30 \%, 50 \%$ and $66 \%$ nitrous oxide also produced a further decrease in amplitude, to Vol. 52, N 3, Maio - Junho, 2002 


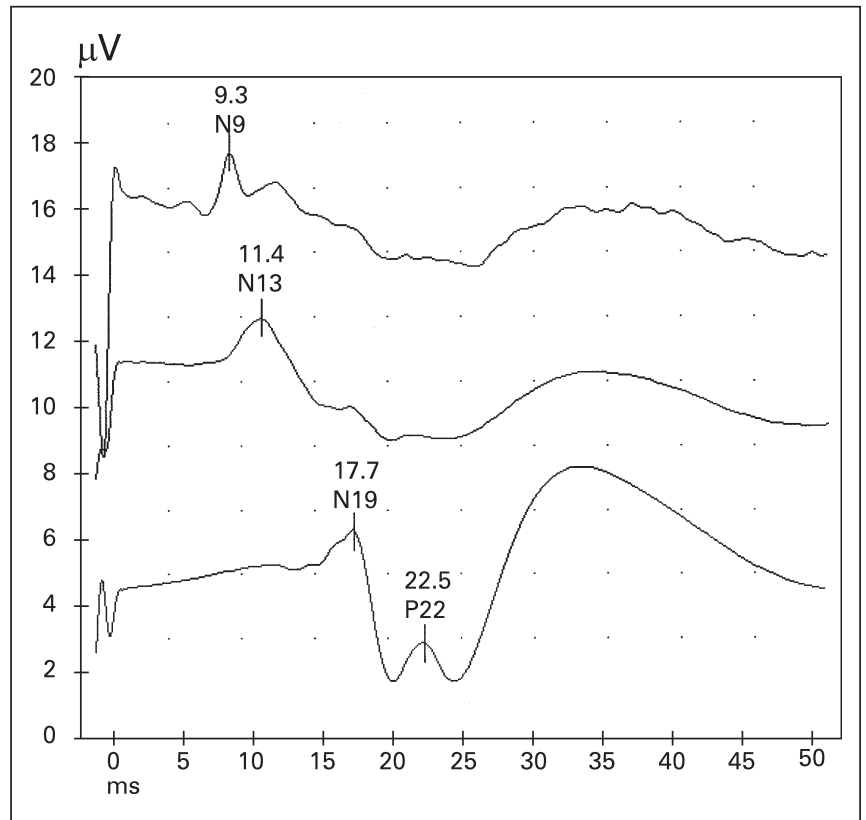

Figure 4 - SEP Waves (N9, N13, N19, P22) Baseline Values (before Nitrous Oxide Administration)

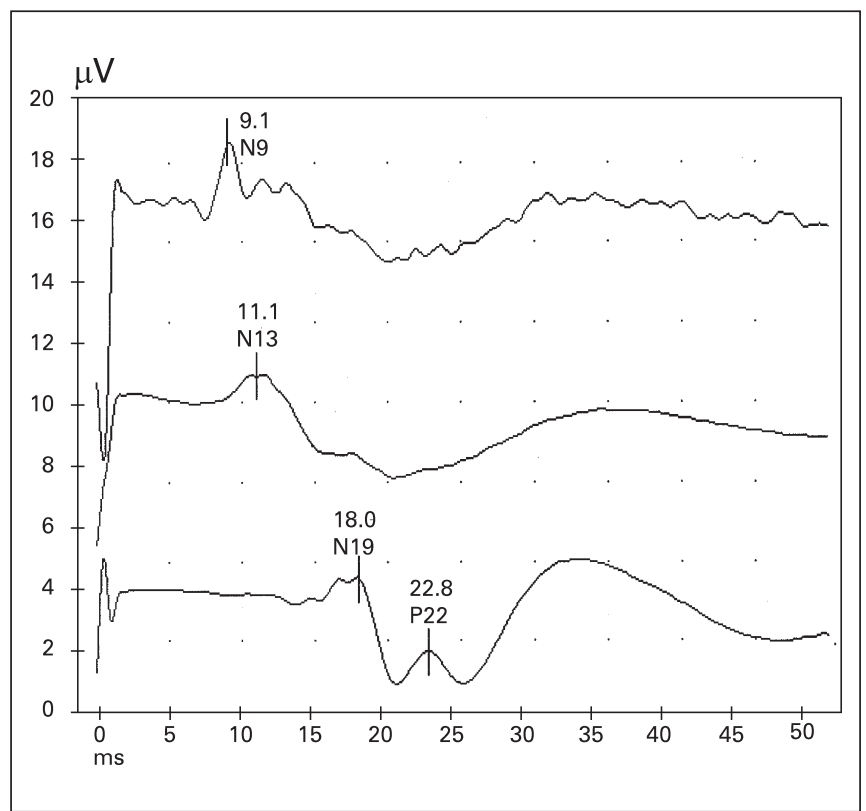

Figure 5 - SEP Waves (N9, N13, N19, P22). Patient with 66\% Nitrous Oxide AF. There is a Minor N13 Amplitude Decrease and a More Significant N19 and P22 Decrease

$48.72 \%(p=0.005), 51.36 \%(p=0.024)$ and $60.50 \%(p=$ $0.010)$, respectively. Onset time increase was intensified when $66 \%$ nitrous oxide was associated $(20.13 \%)$, with no statistical significance though $(p=0.24)$.

Action on Brain Structure - Wave P22:

Nitrous oxide decreased its amplitude in $35.54 \%$ at $30 \% \mathrm{AF}$, $38.52 \%$ at $50 \% \mathrm{AF}$ and $46.48 \%$ at $66 \% \mathrm{AF}$, compared to baseline $(p<0.001)$. There were no changes in onset times (Figure 6).

Isoflurane at $0.5 \mathrm{MAC}$ decreased amplitude in $18.81 \%$, while at $1 \mathrm{MAC}$ this decrease reached $58.59 \%$, compared to baseline, both changes statistically significant $(p=0.017$ and $p=$ 0.014 , respectively). Onset time increased $4.78 \%$ at $0.5 \mathrm{MAC}$ and $14.14 \%$ at $1 \mathrm{MAC}$ compared to baseline. Such changes were not statistically significant though.

Desflurane decreased wave amplitude in $34.13 \%$ at $0.5 \mathrm{MAC}$ and $67.69 \%$ at $1 \mathrm{MAC}$ compared to baseline. This last change (at $1 \mathrm{MAC}$ ) was statistically significant $(p=0.001)$. Onset time increased $7.48 \%$ at $0.5 \mathrm{MAC}$ and $15.99 \%$ at $1 \mathrm{MAC}$ compared to baseline, however with no statistical significance.

Isoflurane at $1 \mathrm{MAC}$ associated to $30 \%, 50 \%$ and $66 \%$ nitrous oxide decreased amplitude even further in $7.16 \%(p=0.15)$, $54.58 \%(p<0.06)$ and $41.80 \%(p<0.001)$ respectively, with statistical significance at $66 \% \mathrm{~N}_{2} \mathrm{O}$ alveolar concentration. Onset time increase was intensified with $30 \%, 50 \%$ and $66 \%$ $\mathrm{N}_{2} \mathrm{O} A F$ in $20.47 \%$ ( $\left.p=0.043\right), 22.05 \%(p=0.013)$ and $27.24 \%(p=0.066)$ respectively (Figures 7 and 8$)$.

The association of $1 \mathrm{MAC}$ desflurane to $30 \%, 50 \%$ and $66 \%$ nitrous oxide decreased amplitude in $27.23 \%(p=0.03)$, $65.65 \%(p=0.05)$ and $67.71 \%(p=0.04)$ respectively. Onset time increase was intensified with $30 \%, 50 \%$ and $66 \% \mathrm{~N}_{2} \mathrm{O}$ AF in $23.91 \%(p=0.005), 27.25 \%(p=0.007)$ and $31.33 \%(p=$ 0.009 ) respectively (Figures 9 and 10).

\section{DISCUSSION}

Physiological results are in line with other authors confirming the stability of cardiopulmonary functions when nitrous oxide was used as a sole agent or associated to other volatile anesthetics in alveolar concentrations of $30 \%, 50 \%$ and $66 \%$. In such concentrations, nitrous oxide does not act on nervous structures able to inhibit or release certain autonomic neurotransmitters which could trigger cardiovascular

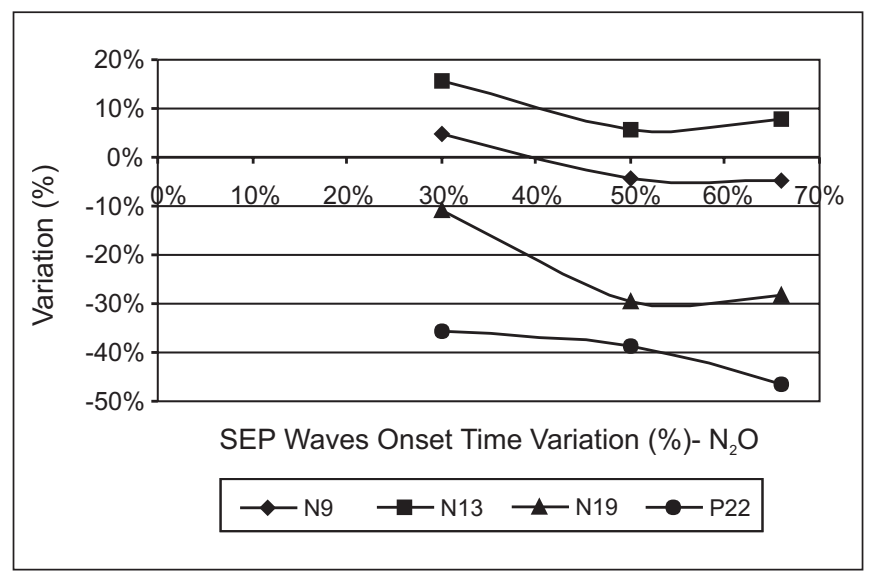

Figure 6 - SEP Waves Amplitude Variation (\%) with Nitrous Oxide as a Sole Agent $(p>0.001)$ Comparison between Baseline Value and $30 \%, 50 \%$ and $66 \%$ after $\mathrm{N}_{2} \mathrm{O}$ Administration 


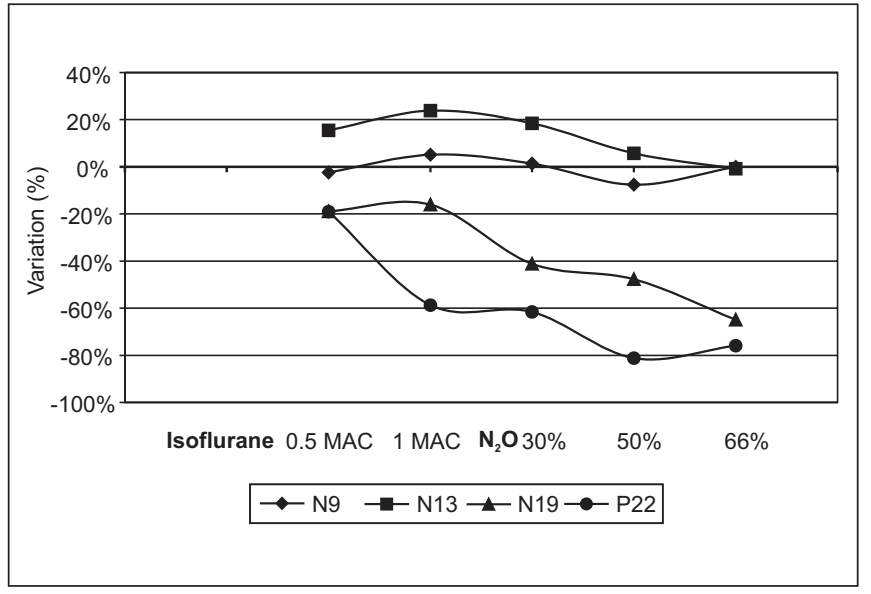

Figure 7 - SEP Waves Amplitude Variation (\%) with Isoflurane as a Sole Agent or Associated to Nitrous Oxide

$(p=0.15$ comparing 1 MAC to 1 MAC associated to $30 \%$ nitrous oxide)

( $p<0.06$ comparing $1 \mathrm{MAC}$ to $1 \mathrm{MAC}$ associated to $50 \%$ nitrous oxide)

( $p<0.001$ comparing $1 \mathrm{MAC}$ to $1 \mathrm{MAC}$ associated to $66 \%$ nitrous oxide)

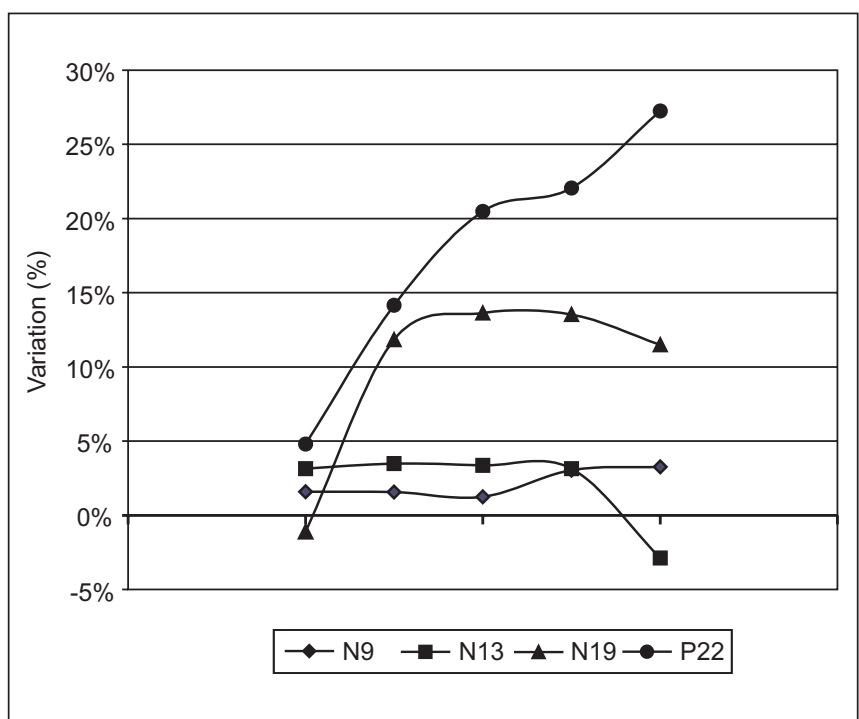

Figure 8 - SEP Waves Onset Time Variation (\%) with Isoflurane as a Sole Agent or Associated to Nitrous Oxide

$(p=0.043$ with $30 \%$ nitrous oxide AF; $p=0.013$ with $50 \%$ nitrous oxide $A F$ e $p=0.066$ with $66 \%$ nitrous oxide $A F$ )

changes, or even inhibit respiratory automation causing alveolar hypoventilation ${ }^{12,14,15}$.

Minor BIS changes with nitrous oxide indicate its very mild hypnotic action.

In our study, mean BIS value dropped from baseline 97.12 to 74.00 when $30 \%$ nitrous oxide was administered. This value kept almost constant (76.64 and 77.64 ) when $50 \%$ and $66 \%$ nitrous oxide were administered.

There are reports in the literature indicating that BIS between 60 and 80 is associated to increased consciousness ${ }^{16}$. So al-

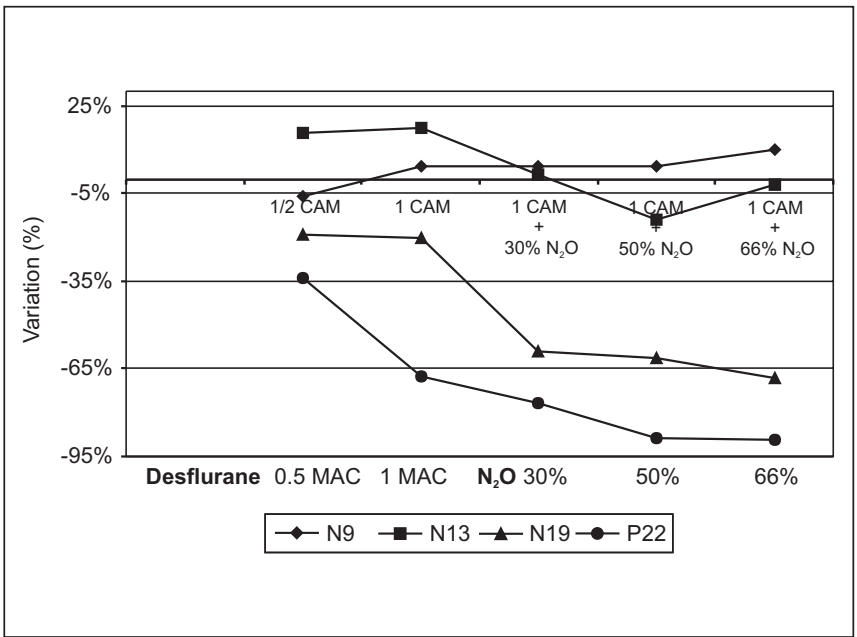

Figure 9 -SEP Waves Amplitude Variation (\%) with Desflurane as a Sole Agent or Associated to Nitrous Oxide

$(p=0.03$ with $30 \%$ nitrous oxide AF; $p=0.05$ with $50 \%$ nitrous oxide AF e $p=0.04$ with $66 \%$ nitrous oxide AF)

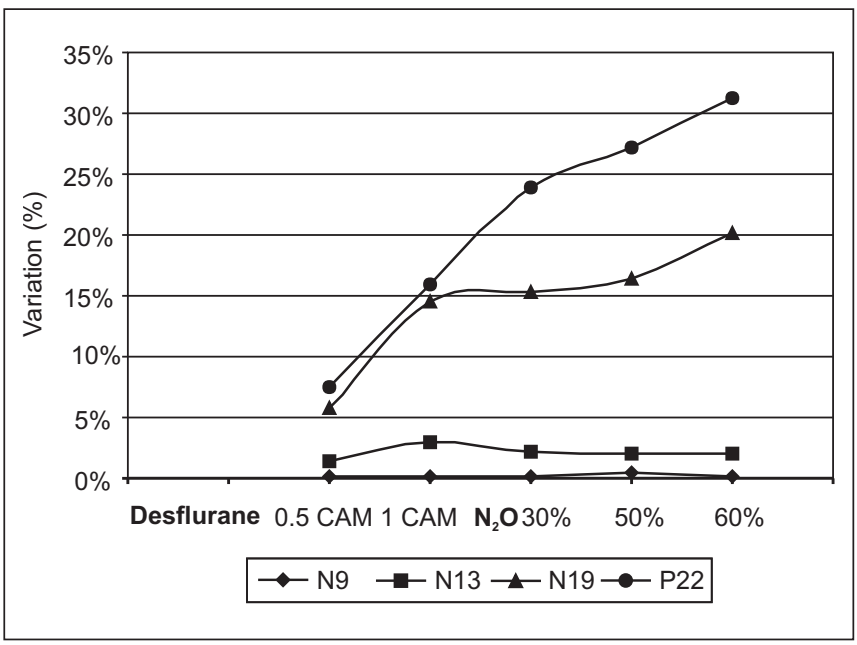

Figure 10 -SEP Waves Onset Time Variation (\%) with Desflurane as a Sole Agent or Associated to Nitrous Oxide $(p=0.005$ with $30 \% \mathrm{AF} ; \mathrm{p}=0.007$ with $50 \% \mathrm{AF}$ and $\mathrm{p}=0.009$ with $66 \%$ nitrous oxide AF)

though decreased, values reached in all studied concentrations did not indicate a total hypnotic state. The initial decrease with $30 \%$ nitrous oxide could be related to residual intravenous propofol effect used for anesthetic induction. However, BIS decrease after a propofol bolus injection is much higher, indicating total hypnosis, and it takes only 8 minutes for it to increase above 60 , followed by consciousness return ${ }^{17}$. In our study, after propofol induction, there was a 30-minute interval when no anesthetics were used. BIS did not return to baseline, but reached approximately 80 . This value is clearly closer to alertness than to hypnosis. Studies have shown that patients receiving 10\%, 20\%,30\%, $40 \%$ and $50 \%$ nitrous oxide had no BIS change ${ }^{18}$. Another study has

Revista Brasileira de Anestesiologia Vol. 52, № 3, Maio - Junho, 2002 
shown that, even with $70 \%$ nitrous oxide, there have been no significant BIS change that could characterize hypnosis ${ }^{19}$. Isoflurane and desflurane decrease BIS in a dose-dependent way. This shows that different from nitrous oxide those agents have an important hypnotic action. Other author has also demonstrated the same results for drugs such as propofol, midazolam and isoflurane ${ }^{20}$. There are reports on BIS increase with high isoflurane concentrations ${ }^{21}$. Our study showed no BIS increase with increased isoflurane concentrations, but we did not exceed 1 MAC. Maybe this limited concentration would justify this difference in results. Regarding desflurane, there is no report in the literature using concentrations similar to ours.

The association of nitrous oxide to $1 \mathrm{MAC}$ isoflurane and desflurane promoted a mild BIS decrease, never beyond $10 \%$ compared to values obtained without this gas. The decrease was a little greater in patients receiving desflurane. These results are in line with a previous study where the author has found no difference in BIS when nitrous oxide was used a sole agent $(70 \%)$ or associated to isoflurane, in spite of consciousness loss ${ }^{19}$. Another author has reported that $50 \%$ nitrous oxide as sole agent caused mild sedation and no BIS change. It was, however, observed by EEG activation in certain areas, which did not affect BIS, indicating that nitrous oxide has CNS inhibitory and excitatory effects ${ }^{18}$.

There are studies showing different nitrous oxide effects on CNS, some of which not in agreement with results found in our study. It has been reported that nitrous oxide antagonizes isoflurane depressing effects on EEG ${ }^{922}$. Isoflurane would produce a higher BIS value associated to nitrous oxide than when employed as sole agent ${ }^{23}$. Another study has shown that the addition of nitrous oxide increased a propofol induced low BIS, although the patients continued not to respond to verbal commands ${ }^{24}$, probably due to amnesia. In fact, studies about nitrous oxide effects on BIS are conflicting, possibly reflecting an inadequate algorithm used to calculate BIS from EEG. These findings question BIS efficiency as anesthetic depth monitor in patients receiving nitrous oxide as a sole agent or associated to other anesthetic agents. In the concentrations used in this study, nitrous oxide did not inhibit neither peripheral nervous structures, such as brachial plexus, nor spinal cord. It is possible that there might has been some action on those structures, especially spinal cord, which was not captured by SEP waves N9 and N13 due to its low magnitude. One must considered that the highest concentration used corresponded to approximately 0.6 MAC of this agent. Usually, concentrations producing even mild inhibition on the spinal cord with other agents are MAC multiples (1.3 to $1.5 \mathrm{MAC}$ ).

Isoflurane and desflurane had also no effect on spinal cord and peripheral potentials. Their action over the stimuli conduction through peripheral nerves and spinal cord seems to be very mild and not enough to affect potentials generated in the brachial plexus and at C2 level.

These findings are similar to other results where the author has not found nitrous oxide-induced changes in subcortical potentials ${ }^{9}$. In another study, the author has described a non-significant change in SEP wave N13 amplitude with nitrous oxide as sole agent or associated to isoflurane. This author, however, agrees that anesthesia has a minor effects on cervical (N13) and brachial plexus (N9) components, and that his results are not in accordance with previous observations ${ }^{25}$. It is suggested that waves N9 and N13 responses are dependent on axonal conduction rather than synaptic transmission, being such potentials less influenced by general anesthesia $^{26}$.

Nitrous oxide inhibits ascending stimuli directed to the brain. This inhibition can be observed as they enter the brain structure, on evoked wave N19, as well as in the cortex by wave P22 inhibition. There was a significant decrease in these waves' amplitude.

Wave N19 showed no onset time change. Amplitude decrease was seen in all $\mathrm{N}_{2} \mathrm{O}$ concentrations, being more pronounced with $50 \%$. There was also no onset time change in wave P22, and amplitude was decreased in $38.52 \%$ with $50 \%$ nitrous oxide. At $66 \% \mathrm{AF}$, amplitude decrease reached $46.48 \%$, with a $12.95 \%$ further reduction compared to previous value (AF 50). It is worth remembering that amplitude decrease was not exacerbated in wave $\mathrm{N} 19$ when $\mathrm{N}_{2} \mathrm{O}$ AF changed from $50 \%$ to $66 \%$. Some authors have described nitrous oxide-induced amplitude decrease without changes in cortical waves onset time ${ }^{10,25,27}$. However, none of those studies has shown so close percentual changes in N19 wave amplitude with different $\mathrm{N}_{2} \mathrm{O}$ concentrations, like our study with $50 \%$ and $66 \%$ AF. And this is the case too for very similar P22 amplitude values with the same $\mathrm{N}_{2} \mathrm{O}$ concentrations. Maybe this was not seen in other studies because the methods were not designed for such observation: the authors have studied just one concentration, or have studied $50 \%$ AF as maximum nitrous oxide concentration.

There is a report in the literature showing that nitrous oxide depressing effect on cortical waves amplitude, evaluated by SEP, is more pronounced than isoflurane's ${ }^{9,25}$. In our study, cortical wave P22 decreased more with 30\%, 50\% and $66 \%$ nitrous oxide than with $0.5 \mathrm{MAC}$ isoflurane. Depression in wave $\mathrm{N} 19$ caused by $50 \%$ and $66 \%$ nitrous oxide was greater than that found with 0.5 and $1 \mathrm{MAC}$ isoflurane, suggesting that nitrous oxide, in equipotent MAC doses, has a higher primary cortex depressing effect than isoflurane. This may explain the good analgesic and amnesic effect of nitrous oxide, as opposed to its weak hypnotic effect. The stimulation of subcortical endogen opioid production and the direct cortical depressing effect were proposed as mechanisms responsible for this analgesic effect 28 . With desflurane, brain waves amplitude reduction with 0.5 MAC was lower than those produced by $50 \%$ and $66 \%$ nitrous oxide. No study was found in the literature comparing nitrous oxide to desflurane, but according to our results, in equipotent MAC doses, nitrous oxide cortical depressing action is stronger than 0.5 MAC desflurane. This fact also reflects the mechanisms responsible for the good analgesic and amnesic effect of such agent. 
The addition of nitrous oxide to isoflurane and desflurane produced significant changes, especially in waves N19 and P22 amplitudes, with minor onset time change of the latter. These results are in line with other authors who have shown that the association of $60 \%$ nitrous oxide significantly decreases cortical waves amplitude ${ }^{28-31}$. In our study, the association of nitrous oxide to isoflurane decreased even more brain waves amplitude. Wave N19 amplitude reduction became more intense as higher nitrous oxide AF were administered. In the other hand, maximum P22 decrease was reached at $50 \% \mathrm{~N}_{2} \mathrm{O}$ AF. So, would there be a limit nitrous oxide concentration from which this gas could no further exacerbate amplitude decrease promoted by $1 \mathrm{MAC}$ isoflurane in this wave? With desflurane, waves N19 and P22 showed a steeper decrease between baseline and $50 \%$ nitrous oxide. From this point on, the reduction became negligible (Figures 9 and 10). The increase in $\mathrm{N}_{2} \mathrm{O}$ AF did not result in proportional brain waves depression, what might be attributed to the existence of a "ceiling effect". Obviously new studies are needed in this direction.

Our study found a greater P22 onset time increase when nitrous oxide was associated to $1 \mathrm{MAC}$ isoflurane and desflurane. It has been reported that when $60 \%$ nitrous oxide were associated to 0.6 MAC isoflurane, only a mild additional wave N19 onset time increase was seen, besides that previously promoted by isoflurane itself. The author has found just 1 millisecond onset time increase, which was not clinically significant ${ }^{25}$. Onset time is seldom used as a criteria for perioperative monitoring due to its low sensitivity ${ }^{32}$. However, we observed a further wave P22 onset time increase with $\mathrm{N}_{2} \mathrm{O}$ addition in all studied concentrations. These findings are in agreement with a previous study where the authors have described an even higher cortical waves onset time increase after the addition of nitrous oxide ( $60 \% \mathrm{AF})$ to sevoflurane ${ }^{33}$.

There are reports in the literature on SEP changes caused by $\mathrm{P}_{\mathrm{ET}} \mathrm{CO}_{2}$ variations ${ }^{34}$, hypothermia ${ }^{35}$, hyperthermia ${ }^{36}$ and surgical stimulation ${ }^{27}$. In our study, patients were under epidural anesthesia and had no significant $\mathrm{P}_{\mathrm{ET}} \mathrm{CO}_{2}$ or temperature changes. So, all BIS and SEP changes may be attributed to nitrous oxide, as well as to isoflurane and desflurane at certain moments.

While producing a marked decrease in cortical potentials amplitude, $\mathrm{N}_{2} \mathrm{O}$ almost does not change BIS, being even able to increase it. It is possible that its actions include a cortical-thalamic inhibition enhancement and an excitatory reduction ${ }^{37}$. However, it has already been reported a nitrous oxide-induced activation in certain brain cortical areas captured by $E E G^{38}$, which might be attributed to a direct brain cortex stimulating effect generating spontaneous bursts, parallel to a reduction in sensory information transmission to the cortex by thalamic nucleus blockade. This results in decreased brain waves amplitude. It has also been observed that nitrous oxide-induced stimulation of reticular centers may be responsible for brain area activation seen on EEG ${ }^{39}$.
In conclusion, it was demonstrated that nitrous oxide causes minor changes in spontaneous stimuli reaching brain cortex and captured by EEG. It mildly changes spinal cord and peripheral potentials when used as a sole agent or associated to isoflurane and desflurane. Nitrous oxide significantly decreases cortical potentials amplitude as sole agent or associated to isoflurane and desflurane, reflecting in its strong analgesic and weak hypnotic effect.

\section{ACKNOWLEDGEMENTS}

The authors acknowledge the neurophysiology team: Alexandre Cardoso Almeida, MD and Maria Dorvalina Silva, MD, and the technicians Josias Francisco de Macedo, Joselda Mara Vieira Lobo, Raimunda Nonata Melo Rosendo and Renata Rodrigues dos Santos, from Hospital Sarah, Brasilia; and especially the statistician Marcio Correa de Mello for supporting this study.

\section{REFERÊNCIAS - REFERENCES}

01. Hornbein TF, Eger EI II, Winter PM et al - The minimum alveolar concentration of nitrous oxide in man. Anesth Analg, 1982;61: 553-556.

02. Eger II EI, Brandstater B, Saidman LJ et al - Equipotent alveolar concentrations of methioxyflurane, halothane, diethyl ether, fluoxene, cyclopropane, xenon and nitrous oxide in the dog. Anesthesiology, 1965;26:771-777.

03. Zancy JP - Time course of effects of brief inhalations of nitrous oxide in normal volunteers. Addiction, 1994;89:831-839.

04. Frost E - A History of Nitrous Oxide, em Eger II EI - Nitrous Oxide/ $\mathrm{N}_{2} \mathrm{O}$. New York, Elsevier, 1985;1-22.

05. Eger II EI, Larson Jr CP - Anaesthetic solubility in blood and tissues: values and significance. Br J Anaesth, 1964;36:140-149.

06. Saidman LJ, Eger II El- Effect of nitrous oxide and of narcotic premedication on the alveolar concentration of halothane required for anesthesia. Anesthesiology, 1964;25:302-306.

07. Thornton JA, Fleming JS, Goldberg AD et al - Cardiovascular effects of $50 \%$ nitrous oxide and $50 \%$ oxygen mixture. Anaesthesia, 1973;28:484-489.

08. Eger II EI - MAC, em: Eger II EI - Nitrous Oxide/ $\mathrm{N}_{2} \mathrm{O}$. New York, Elsevier, 1985:57-67.

09. Porkkala T, Jäntti V, Kaukinen S et al - Nitrous oxide has different effects on the EEG and somatosensory evoked potentials during isoflurane anesthesia in patients. Acta Anaesthesiol Scand, 1997;41:497-501.

10. Sebel PS, Flynn PJ, Ingram DA - Effect of nitrous oxide on visual, auditory and somatosensory evoked potentials. Br J Anaesth, 1984;56:1403-1407.

11. Clark DL, Rosner BS - Neurophysiologic effects of general anesthetics: I. The electroencephalogram and sensory evoked responses in man. Anesthesiology, 1973;38:564-582.

12. Horkey J, Bustillo F, Norton ML - Evaluation of the effects of nitrous oxide in man. Anesth Prog, 1973;20:16-19.

13. Levy WJ - Effect of epoch length on power spectrum analysis of the EEG. Anesthesiology, 1987;66:489-495.

14. Eger II El, Lampe GH, Wauk LZ et al - Clinical pharmacology of nitrous oxide: an argument for its continued use. Anesth Analg, 1990;71:575-585. 
15. Eger II EI - Respiratory Effects of Nitrous Oxide, em: Eger II EI Nitrous Oxide/ $\mathrm{N}_{2} \mathrm{O}$.New York, Elsevier, 1985:109-123.

16. Kearse L, Rosow P, Sebel P et al - The bispectral index correlates with sedation/hypnosis and recall: comparison using multiple agents. Anesthesiology, 1995;83:A507.

17. Flaishon R, Windsor A, Sigl J et al - Recovery of consciousness after thiopental or propofol. Bispectral index and the isolated forearm technique. Anesthesiology, 1997;86:613-619.

18. Rampil IJ, Kim J, Lenhardt R et al - Bispectral EEG index during nitrous oxide administration. Anesthesiology, 1998;89:671-677.

19. Barr G, Jakobsson JG, Öwall A et al - Nitrous oxide does not alter bispectral index: study with nitrous oxide as sole agent and as an adjunct to i.v. anaesthesia. Br J Anaesth, 1999;82:827-830.

20. Glass PS, Bloom M, Kearse L et al - Bispectral analysis measures sedation and memory effects of propofol, midazolam, isoflurane, and alfentanil. Anesthesiology, 1997;86:836-847.

21. Detsch O, Schneider G, Kochs $E$ et al - Increasing isoflurane concentration may cause paradoxical increases in the EEG bispectral index in surgical patients. $\mathrm{Br} \mathrm{J}$ Anaesth, 2000;84: 33-37.

22. Yli-Hankala A, Lindgren L, Porkkala T et al - Nitrous oxide-mediated activation of the EEG during isoflurane anaesthesia in patients. Br J Anaesth, 1993;70:54-57.

23. Sebel P, lang E, Rampil L et al - A multicenter study of bispectral encephalogram analysis for monitoring anesthetic effect. Anesth Analg, 1997;84:891-899.

24. Kearse LA, Rosow C, Zaslavsky A et al - Bispectral analysis of the electroencephalogram predicts conscious processing of information during propofol sedation and hypnosis. Anesthesiology, 1998;88:25-34.

25. Lam AM, Sharar SR, Mayberg TS et al - Isoflurane compared with nitrous oxide anaesthesia for intraoperative monitoring of somatosensory-evoked potentials. Can J Anaesth, 1994;41:295-300.

26. Samra SK, Vanderzant CW, Domer PA et al - Differential effects of isoflurane on human median nerve somatosensory evoked potentials. Anesthesiology, 1987;66:29-35.

27. Sloan TB, Koht A-Depression of cortical somatosensory evoked potentials by nitrous oxide. Br J Anaesth, 1985;57: 849-852.

28. Thornton C, Creagh-Barry P, Jordan C et al - Somatosensory and auditory evoked responses recorded simultaneously: differential effects of nitrous oxide and isoflurane. $\mathrm{Br} J$ Anaesth, 1992;68:508-514.

29. Pathak KS, Ammadio M, Kalamchi A et al - Effects of halothane, enflurane, and isoflurane on somatosensory evoked potentials during nitrous oxide anesthesia. Anesthesiology, 1987:66: 753-757.

30. Peterson DO, Drummond JC, Todd MM - Effects of halothane, enflurane, isoflurane, and nitrous oxide somatosensory evoked potentials in humans. Anesthesiology 1986;65:35-40.

31. Schindler E, Muller M, Zickmann B et al - Modulation of somatosensory evoked potentials under various concentrations of desflurane with and without nitrous oxide. J Neurosurg Anesthesiol, 1998;10:218-223.

32. Lam AM, Manninen PH, Ferguson GG et al - Monitoring electrophysiological function during carotid endarterectomy: a comparison of somatosensory evoked potentials and conventional EEG. Anesthesiology, 1991;75:15-21.

33. Costa VV, Saraiva RA, Almeida AC et al - The effect of nitrous oxide on the inhibition of somatosensory evoked potentials by sevoflurane in children. Anaesthesia, 2001;56:202-207
34. Mason DG, Higgins D, Boyd SG et al - Effects of isoflurane anaesthesia on the median nerve somatosensory evoked potential in children. Br J Anaesth, 1992;69:562-566.

35. Stejaskal L, Traunicek K, Sourek V et al - Somatosensory evoked potentials in deep hypothermia. Appl Neurophysiol, 1980;43:1-7

36. Dubois M, Coppola R, Buchsbaum MS et al - Somatosensory evoked potential during whole body hyperthermia in human. Electroencephalogr Clin Neurophysiol, 1981;52:157-162.

37. Angel A - Central neuronal pathways and the process of anaesthesia. Br J Anaesth, 1993;71:148-163.

38. Roald OK, Forsman M, Heier MS et al - Cerebral effects of nitrous oxide when added to low and high concentrations of isoflurane in the dog. Anesth Analg, 1991;72:75-79.

39. Algotsson L, Messeter K, Rosén I et al - Effects of nitrous oxide on cerebral haemodynamics and metabolism during isoflurane anaesthesia in man. Acta Anaesthesiol Scand, 1992;36:46-52.

\section{RESUMEN}

Costa VV, Saraiva RA - Acción del Óxido Nitroso en el Sistema Nervioso Central. Estudio Eletrofisiológico como Agente Único y como Agente Coadyuvante

Justificativa y Objetivos - El óxido nitroso es el agente anestésico inhalatorio más utilizado en todo el mundo. Su mecanismo de acción es bastante discutido, con base en resultados experimentales y en evidencias clínicas. El objetivo de este estudio es evaluar la acción eletrofisiológica de este fármaco en el Sistema Nervioso Central a través de monitorización específica.

Método - Fueron estudiados veinticinco pacientes de ambos sexos, con edades entre 6 y 25 años, sometidos a cirugía ortopédica o plástica reparadora, los cuales fueron monitorizados con índice bispectral del eletroencefalograma (BIS) y potencial evocado somatosensitivo (PESS) durante la anestesia. Fueron realizados registros basales del BIS y PESS, bien como después del uso del óxido nitroso en fraccionales alveolares (FA) de $30 \%, 50 \%$ y $66 \%$. En seguida el óxido nitroso era descontinuado y administrado aleatoriamente isoflurano o desflurano en 0,5 CAM y 1 CAM. Se mantenía 1 CAM del determinado agente y el óxido nitroso era nuevamente administrado en las mismas concentraciones anteriores.

Resultados - El óxido nitroso cuando utilizado como agente único, produce una reducción en el BIS que, aunque sea estadísticamente significante, no expresa un estado de hipnosis. Esta reducción también ocurre cuando utilizado como agente coadyuvante más sin importancia clínica. Como agente único, el óxido nitroso deprimió significantemente la amplitud de las ondas cerebrales, sin promover aumento en la latencia. El isoflurano y desflurano redujeron la amplitud y aumentaron la latencia de las ondas cerebrales. La asociación del óxido nitroso a estos agentes, intensificó aun más estos efectos en las ondas corticales. No hubo alteración significativa de las ondas periférica y medular del PESS.

Conclusiones - El óxido nitroso tiene una pequeña acción hipnótica, que no es captada completamente por el BIS. Tiene acción acentuada en las estructuras corticales, tanto como agente único como asociado al isoflurano y desflurano, lo que puede explicar su buen efecto analgésico. 